\title{
Tau mutant A152T, a risk factor for FTD/ PSP, induces neuronal dysfunction and reduced lifespan independently of aggregation in a C. elegans Tauopathy model
}

Ghulam Jeelani Pir ${ }^{1,3^{*}+}$, Bikash Choudhary ${ }^{1,3+}$, Eckhard Mandelkow ${ }^{1,2,3}$ and Eva-Maria Mandelkow ${ }^{1,2,3^{*}}$

\begin{abstract}
Background: A certain number of mutations in the Microtubule-Associated Protein Tau (MAPT) gene have been identified in individuals with high risk to develop neurodegenerative diseases, collectively called tauopathies. The mutation A152TMAPT was recently identified in patients diagnosed with frontotemporal spectrum disorders, including Progressive Supranuclear Palsy (PSP), Frontotemporal Dementia (FTD), Corticobasal Degeneration (CBD), and Alzheimer disease (AD). The A152TMAPT mutation is unusual since it lies within the N-terminal region of Tau protein, far outside the repeat domain that is responsible for physiological Tau-microtubule interactions and pathological Tau aggregation. How A152TMAPT causes neurodegeneration remains elusive.
\end{abstract}

Results: To understand the pathological consequences of this mutation, here we present a new Caenorhabditis elegans model expressing the mutant A152TMAPT in neurons. While expression of full-length wild-type human tau $\left(T_{a}{ }^{\mathrm{wt}}, 2 \mathrm{~N} 4 \mathrm{R}\right)$ in C. elegans neurons induces a progressive mild uncoordinated locomotion in a dose-dependent manner, mutant tau (Tau ${ }^{\mathrm{A} 152 \mathrm{~T}}, 2 \mathrm{~N} 4 \mathrm{R}$ ) induces a severe paralysis accompanied by acute neuronal dysfunction. Mutant Tau ${ }^{\mathrm{A} 152 \mathrm{~T}}$ worms display morphological changes in neurons reminiscent of neuronal aging and a shortened life-span. Moreover, mutant A152T overexpressing neurons show mislocalization of pre-synaptic proteins as well as distorted mitochondrial distribution and trafficking. Strikingly, mutant tau-transgenic worms do not accumulate insoluble tau aggregates, although soluble oligomeric tau was detected. In addition, the full-length A152T-tau remains in a pathological conformation that accounts for its toxicity. Moreover, the $\mathrm{N}$-terminal region of tau is not toxic per se, despite the fact that it harbours the A152T mutation, but requires the C-terminal region including the repeat domain to move into the neuronal processes in order to execute the pathology.

Conclusion: In summary, we show that the mutant Tau ${ }^{\mathrm{A} 152 \mathrm{~T}}$ induces neuronal dysfunction, morphological alterations in neurons akin to aging phenotype and reduced life-span independently of aggregation. This comprehensive description of the pathology due to Tau ${ }^{A 152 T}$ opens up multiple possibilities to identify cellular targets involved in the Tau-dependent pathology for a potential therapeutic intervention.

Keywords: AD, Alzheimer disease, PSP, Progressive supranuclear palsy, FTD, Frontotemporal dementia, CBD, Corticobasal degeneration

\footnotetext{
* Correspondence: Jeelani.Pir@dzne.de; eva.mandelkow@dzne.de

${ }^{\dagger}$ Equal contributors

${ }^{1}$ German Center for Neurodegenerative Diseases (DZNE), Ludwig-Erhard-Allee

2, 53175 Bonn, Germany

Full list of author information is available at the end of the article
} 


\section{Background}

Neurodegenerative diseases are a leading cause of dementia and pose a serious threat to our aging population. Among them, tauopathies (including $\mathrm{AD}$ and FTDP-17) characterized by the accumulation of highly phosphorylated insoluble tau [1-3] are the most common. Tauopathies can be of two types [4]; sporadic and familial. A small percentage of patients (1-5\%) develop disease as a result of mutations in the tau gene (MAPT). These mutations can have multifarious consequences; they are capable of reducing the microtubule binding affinity of tau and therefore result in a partial loss of function of tau [5]. Moreover, these mutations are able to increase the beta-propensity of tau, making it more prone to aggregation. Consistent with this, the majority of MAPT mutations studied so far are clustered in or near the repeat domain of tau which is responsible for aggregation [1]. However, a rare point mutation in tau gene (A152T) was recently reported in patients suffering from PSP, FTD, or AD [6-8]. This mutation is unique since it lies far outside of the repeat domain in the $\mathrm{N}$ terminal proline-rich region which is thought to interact with different signalling pathways due to its interactions with proteins containing SH3-domains $[9,10]$.

The amino acid substitution A152T in tau has been shown in vitro to be associated with a reduced microtubule binding affinity and, an increase in formation of Tau oligomers instead of insoluble PHFs [6]. Whether this amino acid substitution has any bearing on the development of disease remains uncertain, due to scarcity of in vivo data. Therefore, to understand the pathological consequences of the A152T mutation in the context of a whole organism, we used the nematode C. elegans as a model. It offers several advantages over other animal models. For example, it has a transparent body with a simple nervous system, which makes it possible to image the progression of pathology at the cellular and sub-cellular level. It has a short life-span (2-3 weeks) and is genetically well characterized and tractable. For these reasons, C. elegans has been extensively used as a model of neurodegeneration [11-13] and has provided invaluable insights into the nature of the pathology involved. Several key pathways and signalling molecules are conserved between worms and mammals [14]. Two genes implicated in tau pathology (sut-1 and sut-2) have been identified in a genetic screen using $C$. elegans $[15,16]$, which point to effects involving the microtubule and actin cytoskeleton. More recently, we demonstrated the potential of using C. elegans as a tool for the screening of neuroprotective compounds; in fact one of the compounds (MB) that proved beneficial to this model [17] is running in the $3^{\text {rd }}$ phase of clinical trial [18]. All in all, studies of the $C$. elegans nervous system have greatly aided efforts to analyze the causes of neurodegenerative diseases on the path to developing an effective treatment.
To investigate the functional consequences of this mutation in vivo, we generated pan-neuronal mutant A152Ttau (htau40A152T, hereafter referred to $\mathrm{Tau}^{\mathrm{AT}}$ ) and wildtype tau (htau40WT, or $\mathrm{Tau}^{\mathrm{wt}}$ ) overexpressing nematodes. We found that, while the expression of $\mathrm{Tau}^{\mathrm{wt}}$ led only to mild pathology over time and in dose-dependent manner, the mutant Tau ${ }^{\mathrm{AT}}$ showed pronounced pathology from an early age. The pathology induced by $\mathrm{Tau}^{\mathrm{AT}}$ was doseindependent, manifested as severe paralysis, defects in the GABAergic motor neurons, accumulation of morphological abnormalities in touch neurons reminiscent of neuronal aging and reduced life span. At the cellular level, $\mathrm{Tau}^{\mathrm{AT}}$ worms displayed altered distribution of major neuronal organelles (synaptic vesicles and mitochondria) and a perturbed mitochondrial trafficking. The worms expressing $\mathrm{Tau}^{\mathrm{AT}}$ showed the protein in a pathological state as confirmed by staining with the conformation-dependent MC1 antibody, whereas Tau ${ }^{\text {wt }}$ worms showed only minimal staining. Furthermore, in mutant worms Tau ${ }^{\mathrm{AT}}$ appeared mostly as soluble higher molecular weight oligomers, albeit they did not accumulate as insoluble aggregates. We also demonstrated that although the mutation site lies in the N-terminal half of $\mathrm{Tau}^{\mathrm{AT}}$, the Cterminal half which engages with the microtubules is necessary for pathology.

\section{Results \\ Expression of mutant $\mathrm{Tau}^{\mathrm{AT}}$ leads to severe locomotor defects}

To examine the consequences of the Tau mutation A152T in vivo, we generated transgenic worms expressing constitutively either the wild-type $\mathrm{Tau}^{\mathrm{wt}}$ or the Tau ${ }^{\mathrm{AT}}$ mutant form of human 2N4R Tau in neurons. The panneuronal expression was achieved using the synaptobrevin promoter $s n b-1 p$ (Fig. 1a). Multiple lines were obtained after transgene integration. We first compared the protein levels by subjecting the worm lysates from each strain (3 day old adults) to western blotting using K9JA pan-Tau antibody. Two lines from each set of transgenes were selected for further analysis; worms showing comparably lower expression levels (referred to hereafter as Tau ${ }^{\text {wt }}$-lo or $\mathrm{Tau}^{\mathrm{AT}}$-lo, resp.) or higher expression levels (termed $\mathrm{Tau}^{\mathrm{wt}}$-hi and $\mathrm{Tau}^{\mathrm{AT}}$-hi, resp.). Total tau levels in Tau ${ }^{\text {wt }}$-lo and Tau ${ }^{\text {wt }}$-hi lines were comparable with their mutant counterparts $\mathrm{Tau}^{\mathrm{AT}}$-lo and $\mathrm{Tau}^{\mathrm{AT}}$-hi, respectively (Fig. 1b, c). Immunostaining with K9JA antibody showed that Tau transgenes were expressed in the entire nervous system, notably in the major ganglion nerve ring and the nerve cords (Fig. 1d, Additional file 9). Non-transgenic animals did not show any staining for human Tau (data not shown).

Worms expressing Tau ${ }^{\text {wt }}$ exhibit motor defects with mild uncoordinated (Unc) phenotype characterized by sluggishness and a distorted sinusoidal locomotion 


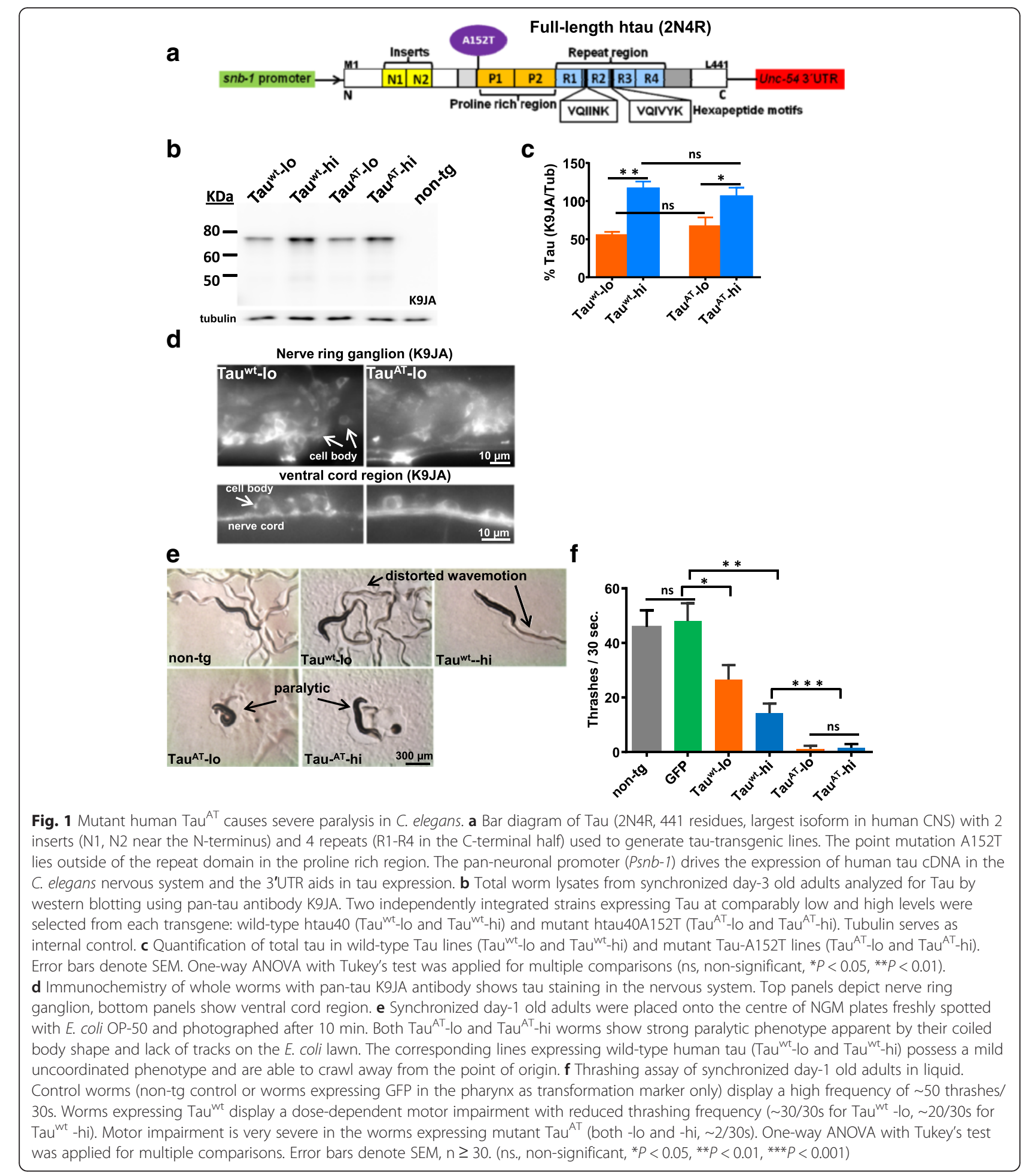

relative to non-transgenic worms (Fig. 1e). However, in worms expressing Tau ${ }^{\mathrm{AT}}$ the severe Unc phenotype is much more pronounced than in worms expressing $\mathrm{Tau}^{\mathrm{wt}}$. Most of the worms expressing mutant Tau ${ }^{\mathrm{AT}}$ were paralytic and were often seen in the coiled state, almost completely unable to move (Fig. 1e). The extent of motor dysfunction in worms expressing $\mathrm{Tau}^{\mathrm{wt}}$ correlated with the protein expression levels, Tau ${ }^{\mathrm{wt}}$-lo with lower tau levels showed a milder phenotype ( 30 thrashes/30 s) than $\mathrm{Tau}^{\mathrm{wt}}$-hi line $(\sim 20$ thrashes/30 s) with higher tau levels (Fig. 1f, also see Additional file 1: Movie S1 and Additional file 2: Movie S2). By contrast, Tau ${ }^{\mathrm{AT}}$-induced 
toxicity did not correlate with protein expression levels; the Unc phenotype was equally severe in $\mathrm{Tau}^{\mathrm{AT}}$-lo and $\mathrm{Tau}^{\mathrm{AT}}$-hi worms (Fig. 1f, also see Additional file 3: Movie S3 and Additional file 4: Movie S4). Tau transgenes ( $\mathrm{Tau}^{\mathrm{wt}}$ and $\mathrm{Tau}^{\mathrm{AT}}$ ) when expressed as extrachromosomal arrays showed comparable Tau levels as detected by western blot using K9JA antibody (see Additional file 10), and yielded similar phenotypes as the integrated lines (see Additional file 10), ruling out any effects due to unwanted background mutations during integration.

\section{Mutant Tau ${ }^{\mathrm{AT}}$ leads to pronounced GABAergic neurodegeneration, while $\mathrm{Tau}^{\mathrm{wt}}$ causes mild degeneration only at higher levels}

Normal sinusoidal locomotion in C. elegans is made possible by coordinated activity of reciprocal motor circuits comprised of inhibitory GABAergic and excitatory cholinergic neurons located along the body wall [19], and any interruption in the networking produces a defective locomotion (uncoordinated). To evaluate the possibility that the locomotion phenotype in tau-transgenic worms is due to a defective motor nervous system, the integrity of GABAergic motor system was monitored. Animals were analyzed for axonal gaps in the ventral and dorsal cords by crossing them into the juIs73:[Punc-25::gfp]III transgene [20] that allows visualization of GABAergic motor neurons by expression of GFP (Fig. 2b). GABAergic motor neurons in both the worm strains expressing $\mathrm{Tau}^{\mathrm{AT}}$ ( $\mathrm{Tau}^{\mathrm{AT}}$-lo and $\mathrm{Tau}^{\mathrm{AT}}$-hi) were severely abnormal with frequent gaps in ventral and dorsal cords, often showing stretches of nerve cords missing (Fig. 2e, F and see Additional file 10, Additional file 11), consistent with the extent of motor dysfunction shown by these worms (see Fig. 1e). On the other hand, GABAergic motor neurons in worms expressing $\mathrm{Tau}^{\mathrm{wt}}$ were slightly affected (lesser gaps) when compared to worms expressing Tau ${ }^{\mathrm{AT}}$ (Fig. 2c, d; Table 1). However, when compared to non-tg reporter strain (juIs73), $\mathrm{Tau}^{\mathrm{wt}}$ worms did exhibit a significant number of gaps (Table 1). Consistent with the motor deficits, the extent of neuronal damage seen in worms expressing Tau ${ }^{\text {wt }}$ depended on the level of protein expression, with Tau ${ }^{\mathrm{wt}}$-hi accumulating more damage than Tau ${ }^{\text {wt }}$-lo (Table 1 ).

Since the lines $\mathrm{Tau}^{\mathrm{AT}}$-lo and $\mathrm{Tau}^{\mathrm{AT}}$-hi did not differ much in their phenotypes, from hereon we decided to compare the two wild-type tau lines (Tau ${ }^{\text {wt }}$-lo and Tau ${ }^{\text {wt }}$-hi, which differ depending on Tau levels) with the mutant line with lower tau expression ( $\mathrm{Tau}^{\mathrm{AT}}$-lo), unless otherwise mentioned.

\section{Mutant $\mathrm{Tau}^{\mathrm{AT}}$ induces hallmarks of aging neurons and shortens the life-span}

The above microscopical observations of the GABAergic neurons clearly showed that their processes are degenerating, consistent with the locomotor defects seen in
Tau-tg worms. As neuronal restructuring and synaptic disintegration is one of the hallmarks of aging nervous system [21, 22], we asked whether the Tau ${ }^{\mathrm{AT}}$ variant initiates such changes which could be interpreted as indication of premature aging of the nervous system. Mechanosensory neurons (touch neurons) offer an excellent model to study changes associated with neuronal aging in C. elegans. These neurons undergo morphological changes such as irregular outgrowths from somata, extra neurites, and bending of main neuronal processes that become exaggerated with age $[23,24]$. We therefore generated Tau-tg worms carrying $z d I s 5:[$ Pmec-4::GFP + lin-15(+)] transgene that specifically express GFP in the six mechanosensory neurons. We observed significant morphological alterations of these neurons early in adulthood (day 1) of $\mathrm{Tau}^{\mathrm{AT}}$ worms, changes that have been associated with aging in previous studies [23, 24]. We focussed on ALM neurons (anterior lateral microtubule cells) located in the anterior body region. ALM neuronal processes in Tau ${ }^{\mathrm{AT}}$ worms appeared wavy, in contrast to the straight processes seen in non-tg $(z d I s 5)$ and $\mathrm{Tau}^{\mathrm{wt}}$ worms (both $\mathrm{Tau}^{\mathrm{wt}}$-lo and Tau ${ }^{\mathrm{wt}}$-hi), and new outgrowths started emanating from the somata already at day 1 (Fig. 3b, panel day1). A higher percentage of $\mathrm{Tau}^{\mathrm{AT}}$-lo worms ( 70\%) showed these morphological abnormalities at day $1 \mathrm{com}$ pared to non-tg ( 12\%), Tau ${ }^{\mathrm{wt}}-\mathrm{lo}(\sim 20 \%)$ and Tau ${ }^{\mathrm{wt}}$-hi worms ( 23\%) (Fig. 3d). Furthermore, nearly $10 \%$ of non-tg animals showed a single posterior projecting neurite emanating from ALM somata at day 1 consistent with the earlier studies [24], whereas the incidence of such neurites in Tau ${ }^{\mathrm{AT}}$-lo ALM neurons was found in almost all the animals. By contrast, worms expressing Tau ${ }^{\mathrm{wt}}$ $\left(\mathrm{Tau}^{\mathrm{wt}}-\mathrm{lo}\right.$ and $\left.\mathrm{Tau}{ }^{\mathrm{wt}}-\mathrm{hi}\right)$ showed normal morphology (Fig. 3b, panel day1). However, the number of animals showing ALM neurons with a single posterior projecting neurite was higher in Tau ${ }^{\mathrm{wt}}$ worms $(\sim 30 \%)$ compared to non-tg animals ( 12\%). The severity of these morphological abnormalities further increased with age, new outgrowths that started emanating from ALM somata of day1 old $\mathrm{Tau}^{\mathrm{AT}}$-lo worms had extended to a considerable length and occasionally underwent branching. Furthermore, the single posterior projecting neurite also showed new outgrowths at multiple sites by day 3 of adulthood (example in Fig. 3c, panel day 3). By this age, about $30 \%$ of the worms expressing Tau ${ }^{\text {wt }}$ showed morphological abnormalities similar to day-1 Tau ${ }^{\mathrm{AT}}$ worms (Fig. 3d).

A similar trend was seen in PLM neurons (posterior lateral microtubule cells), but the degree of restructuring (accumulated damage) was less extensive ( $\mathrm{Tau}^{\mathrm{wt}}-\mathrm{lo}: \sim 10 \%$, $\mathrm{Tau}^{\mathrm{wt}}$-hi $\sim 20 \%$ and Tau ${ }^{\mathrm{AT}}$-lo: $\sim 60 \%$ at day 1$)$. This is consistent with earlier reports whereby neuronal populations were found to differ in terms of vulnerability during aging [24]. Overall our data suggests that the appearance of these morphological changes in neurons that accompany 

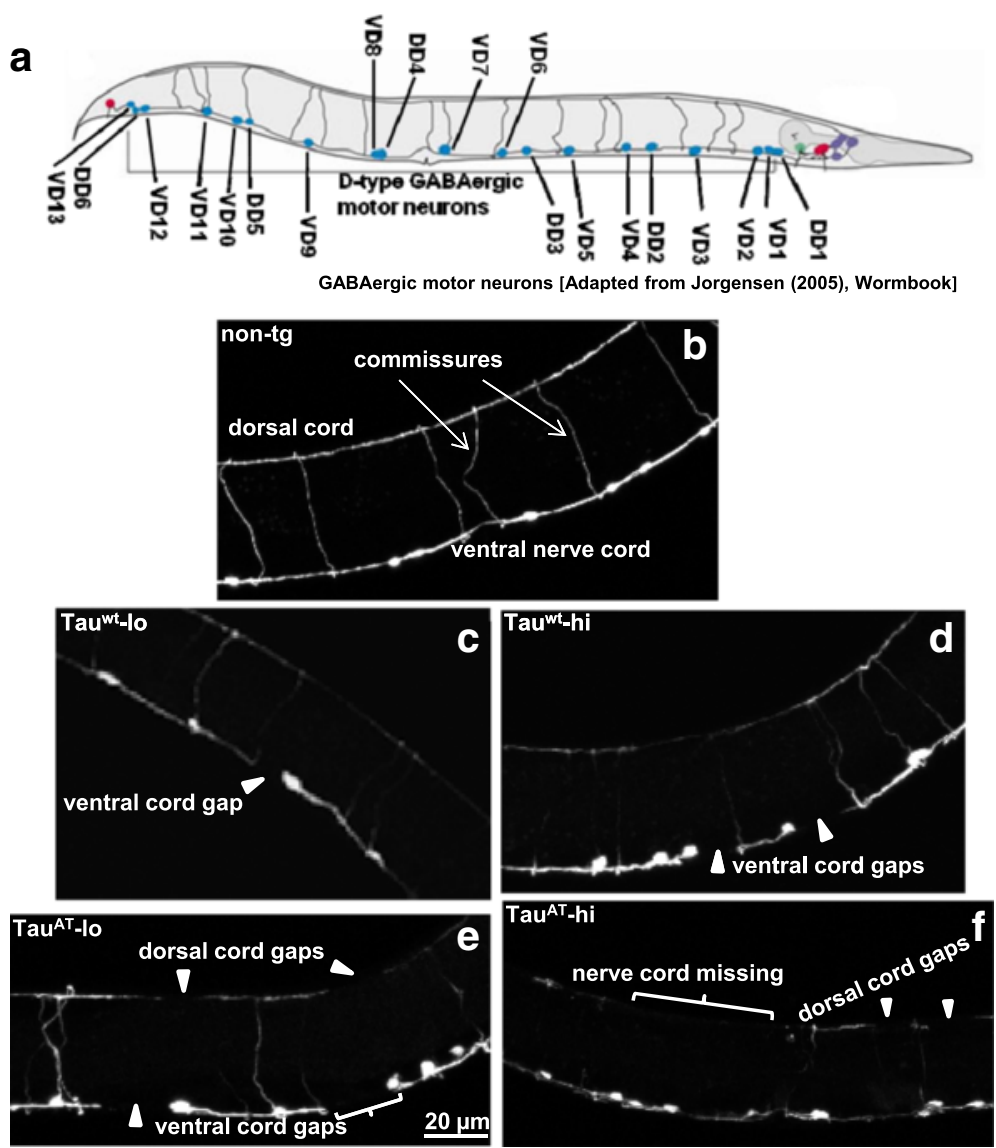

Fig. 2 Mutant Tau ${ }^{A T}$ leads to substantial damage in GABAergic motor neurons. Punc-25::gfp reporter that labels GABAergic inhibitory neurons with GFP, was crossed with respective tau-transgenic worms to visualize these neurons. a Cartoon depicting the GABAergic inhibitory motor system in an adult worm. b-f depict representative maximum intensity projections (MIP) of day-1 old adults of the transgenes. For whole worm MIPs, see Additional file 11. Abnormalities seen as gaps in the GABAergic inhibitory neurons are highlighted by arrowheads. GABAergic neurons show normal connectivity in non-tg reporter worms, both dorsal and ventral nerve cords are intact (b). Expression of Tau ${ }^{\text {wt }}$ produces dose dependent abnormalities in GABAergic neurons, with Tau ${ }^{\text {wt }}$-hi neurons (d) accumulating more damage than Tau ${ }^{\text {wt }}$-lo (c). However, mutant Tau ${ }^{A T}$ expression leads to severe abnormalities in the form of gaps in the dorsal and ventral nerve cords, both Tau ${ }^{A T}-\mathrm{lo}(\mathbf{e})$ and Tau ${ }^{\mathrm{AT}}$-hi $(\mathbf{f})$. One of the striking features of mutant Tau ${ }^{\mathrm{AT}}$ worms is the absence of stretches of dorsal cord (bracketed areas), not found in non-tg worms or Tau ${ }^{\text {wt }}$ worms. See Table 1 for detailed quantitative analysis

the aging of C. elegans occur early in Tau ${ }^{\mathrm{AT}}$ worms compared to $\mathrm{Tau}^{\mathrm{wt}}$ and non-tg. As previous studies have underlined the role nervous system plays in modulating the lifespan in C. elegans [25-29], one would expect a reduced life-span in the worms expressing $\mathrm{Tau}^{\mathrm{AT}}$. Ultimately, we assessed the survival of Tau-tg animals (Fig. 3e): $\mathrm{Tau}^{\mathrm{wt}}$ expression resulted in a shorter life-span ( $45 \%$ shorter vs. non-tg animals), but mutant Tau ${ }^{\mathrm{AT}}$ expression decreased the life-span even much more ( $65 \%$ shorter vs. non-tg, $\sim 25 \%$ shorter vs. Tau ${ }^{\text {wt }}$ (-lo or -hi) (Table 2 ).

\section{Mutant $\mathrm{Tau}^{\mathrm{AT}}$ worms display perturbed distribution of major neuronal organelles and defective presynaptic neurotransmission}

Since $\mathrm{Tau}^{\mathrm{AT}}$ worms showed early morphological alterations mimicking aging neuronal phenotypes, we sought to investigate the status of the neuronal transport
Table 1 Quantification of GABAergic neuronal damage in day-1 old Tau-tg worms

\begin{tabular}{llll}
\hline Strain & VC gaps & DC gaps & N \\
\hline non-tg & $0.4 \pm 0.08$ & $0.2 \pm 0.14$ & 25 \\
Tau ${ }^{\text {Wt }-l o ~}$ & $1.4 \pm 0.17^{*}$ & $1.1 \pm 0.09$ & 20 \\
Tau $^{\text {Wt }}$-hi & $2.6 \pm 0.34^{* *}$ & $1.7 \pm 0.71^{*}$ & 18 \\
Tau $^{\mathrm{AT}}$-lo & $5.8 \pm 1.61^{* * *}$ & $6.1 \pm 1.14^{* * *}$ & 24 \\
Tau $^{\mathrm{AT}}$-hi & $6.1 \pm 0.98^{* * *}$ & $5.5 \pm 1.4^{* * *}$ & 25
\end{tabular}

Morphological abnormalities in GABAergic inhibitory neurons apparent as gaps in the dorsal cord (DC gaps) and ventral nerve cord (VC gaps) were assessed with the Punc-25::gfp reporter. $\mathrm{N}$ denotes the total number of animals scored per strain. Non-tg worms with Punc-25::gfp reporter serve as controls. $P$ values for statistical significance are derived using ANOVA (Kruskal-Wallis test) $\left({ }^{*} P<0.05\right.$ vs non-tg, ${ }^{* *} P<0.01$ vs non-tg, ${ }^{* * *} P<0.001$ vs non-tg) 

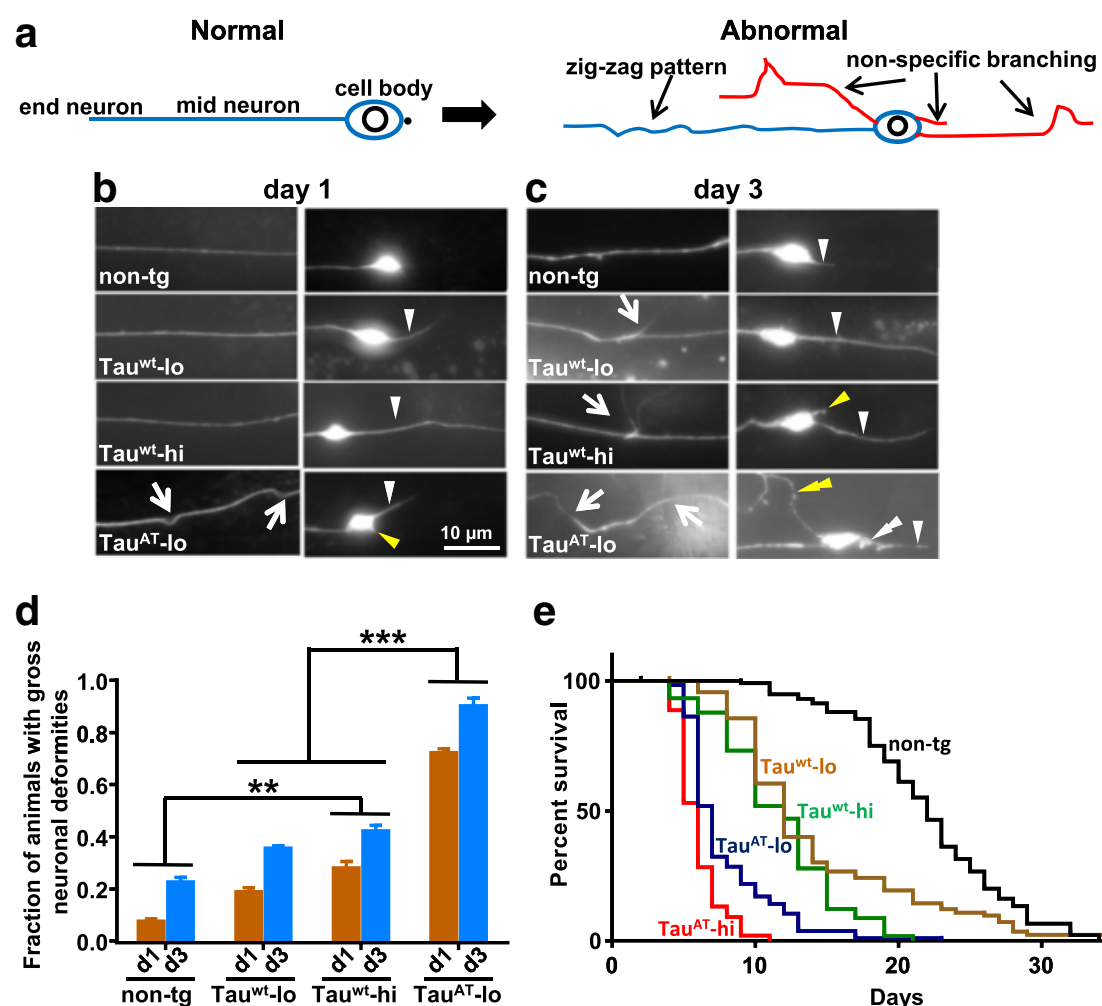

e

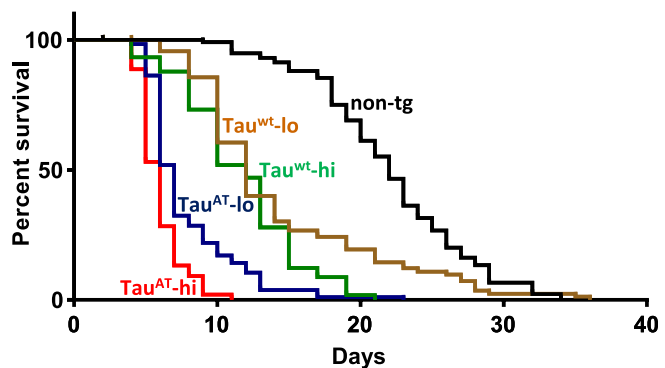

Fig. 3 Mutant Tau ${ }^{\mathrm{AT}}$ induces morphological changes in mechanosensory neurons early in the adulthood and reduces life-span. a Cartoon depicting a normal healthy neuron and a neuron showing morphological changes associated with aging like sprouting, non-specific branching and bending. b Mutant Tau ${ }^{\mathrm{AT}}$ worms show neuronal abnormalities reminiscent of aging neurons. Pmec-4.:-gfp reporter that expresses GFP in mechanosensory neurons, was crossed into tau-transgenic worms and neurons visualized for morphological abnormalities at the days indicated. Note the soma outgrowth (yellow arrowhead) and bending of neuronal process (white arrow) in Tau ${ }^{\mathrm{AT}}$-lo at day 1. At this age, Tau ${ }^{\text {Wt }}$ (both -lo and -hi) show normal morphology and do not differ from non-tg. Only the incidence of an infrequent posterior extension (white arrow heads) increases in the Tau ${ }^{\text {wt }}$ worms (to $30 \%$ compared with $10 \%$ in non-tg at day 1), whereas in Tau ${ }^{\mathrm{AT}}$-lo this posterior extension occurs in almost $100 \%$ of the animals. c The severity of the phenotype increases with age. Soma outgrowths visible in Tau ${ }^{\mathrm{AT}}$-lo animals at day $1 \mathrm{grow}$ and undergo further branching with age (yellow double arrowhead). The volume marker GFP accumulates in beaded structures in the posterior extension in Tau ${ }^{\mathrm{AT}}$-lo worms and small outgrowths emanate from posterior extension (white double arrowhead). These beaded structures could represent starting points of new branches, and were previously shown to be associated with mitochondria [24]. d Quantification of animals with gross non-specific neuronal abnormalities (bends and branches) at two time points, day 1 and day 3. Error bars denote mean \pm SEM $, n \geq 20 .{ }^{* *} P \leq 0.01,{ }^{* * *} P \leq 0.001$. Paired t-test with unequal variance was used for comparison. e Representative survival curves of tau-transgenic animals, non-tg serves as control. Mantel-Cox log-rank test was performed to determine the statistical significance for the worm life-span. (for $P$-values see Table 2)

machinery in the transgenic animals. We checked the distribution of major neuronal cargoes in neurons, which represents an indirect readout for the transport machinery [30]. We first looked at RAB-3, a synaptic vesicle associated Rab GTPase [31], using the the

Table 2 Statistical analysis of life-span assay performed as in Fig. 3e legend

\begin{tabular}{|c|c|c|c|c|c|}
\hline Strain & $\begin{array}{l}\text { Median } \\
\text { survival }\end{array}$ & $\begin{array}{l}\text { \# deaths/ } \\
\text { total N }\end{array}$ & $\begin{array}{l}P \text { value } \\
\text { (vs non-tg) }\end{array}$ & $\begin{array}{l}\text { P value } \\
\text { (vs Tau }\end{array}$ & $\begin{array}{l}P \text { value } \\
\text { (vs Tau }{ }^{\text {wt }} \text {-hi) }\end{array}$ \\
\hline non-tg & 22 & $111 / 120$ & & $>10^{-4}$ & $>10^{-4}$ \\
\hline $\mathrm{Tau}^{\mathrm{wt}}-\mathrm{lo}$ & 12 & $87 / 120$ & $>10^{-4}$ & & $>10^{-4}$ \\
\hline Tau ${ }^{w t}-h i$ & 12 & $67 / 120$ & $>10^{-4}$ & $>10^{-4}$ & \\
\hline $\mathrm{Tau}^{\mathrm{AT}}-\mathrm{lo}$ & 7 & $111 / 120$ & $>10^{-4}$ & $>10^{-4}$ & $>10^{-4}$ \\
\hline $\mathrm{Tau}^{\mathrm{AT}}$-hi & 6 & $110 / 120$ & $>10^{-4}$ & $>10^{-4}$ & $>10^{-4}$ \\
\hline
\end{tabular}

transgene vdEx262:[Pmec-4::mCherry::rab-3;Punc122::gfp] [32] that expresses mCherry fused to RAB-3 in mechanosensory neurons. Worms expressing $\mathrm{Tau}^{\mathrm{wt}}\left(\mathrm{Tau}^{\mathrm{wt}}-\mathrm{lo}\right.$ or Tau ${ }^{\text {wt }}$-hi) showed uniform distribution of RAB-3 along axons similar to non-tg $(v d E \times 262)$ animals at day 1 (Fig. 4b). In contrast, Tau ${ }^{\mathrm{AT}}$-lo worms showed accumulation of RAB-3 in the distal axon and less RAB-3 puncta in the mid-neuron. Furthermore, posterior minor neurites which are usually devoid of RAB-3 (as seen in non-tg worms, $\mathrm{Tau}^{\mathrm{wt}}$-lo and $\mathrm{Tau}^{\mathrm{wt}}$-hi worms), were abnormally filled with RAB-3 clusters in Tau ${ }^{\mathrm{AT}}$-lo worms (Fig. 4b). In older animals, the RAB-3 marker started mislocalizing even in Tau ${ }^{\text {wt }}$ worms such that at day 3, RAB-3 was present in minor neurites, similar to day- 1 old $\mathrm{Tau}^{\mathrm{AT}}$-lo worms (Additional file 12). We quantified the percentage of animals with mislocalized mCherry::RAB-3 puncta in 


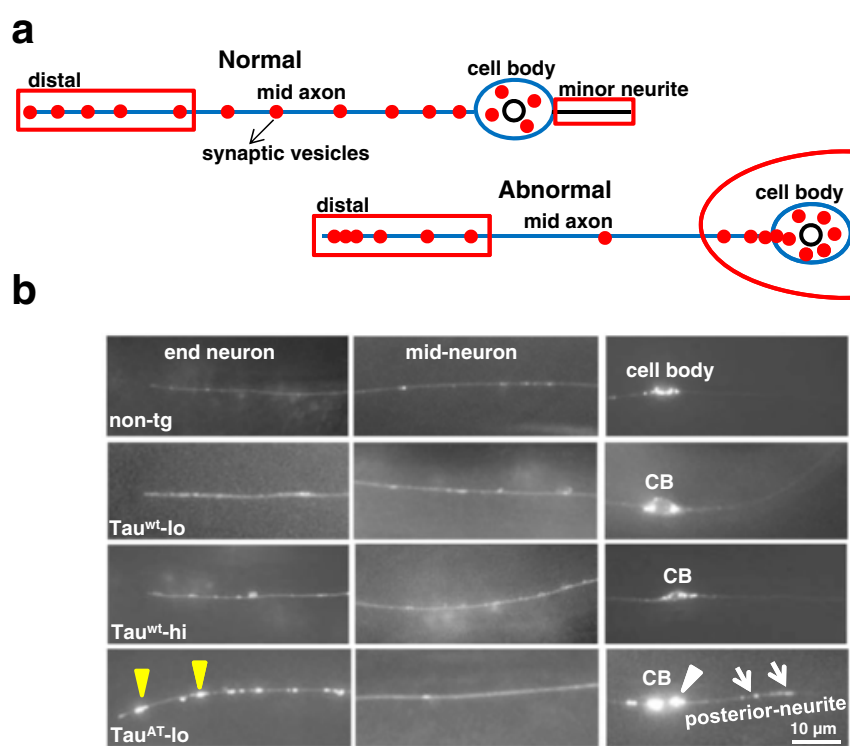

Fig. 4 Mutant Tau ${ }^{\mathrm{AT}}$ worms show aberrant localization of presynaptic components in mechanosensory neurons. a Schematic representation of presynaptic cargo distribution in a normal healthy mechanosensory neuron and a neuron in an aged animal. b Day-1 old worms visualized after crossing them into $v d$ Ex262:[Pmec-4::mCherry::rab-3] transgene that expresses mCherry fused to synaptic vesicle associated RAB-3 in mechanosensory neurons. Tau ${ }^{\text {wt }}$-lo and Tau ${ }^{\text {wt }}$-hi show a similar distribution as non-tg reporter strain. Tau ${ }^{A T}-l o$ worms show accumulation of mCherry::RAB-3 in the end neuron (yellow arrowhead), cell body (CB, white arrowhead) and posterior neurite (white arrow). By contrast, the mid-neuron of Tau ${ }^{A T}$-lo shows less puncta ( $6 \pm 3$ measured per $40 \mu$ m length) than non-tg (15 \pm 5 ) and Tau $\left.{ }^{\text {wt }-l o ~(17 ~} \pm 6\right)$ worms. On day 3 , mislocalization of mCherry:RAB-3 worsens in Tau ${ }^{A T}$-lo worms, whereas Tau ${ }^{\text {wt }}$-lo and Tau ${ }^{\text {wt }}$-hi start accumulating mCherry:RAB-3 puncta in the distal axon and posterior neurite (see Additional file 12)

the distal axon and the minor neurite at day 1 and day 3 . A higher percentage of Tau ${ }^{\mathrm{AT}}$-lo animals ( 60\%) presented mislocalized mCherry::RAB-3 compared to non-tg worms ( 5 \%), Tau ${ }^{\text {wt }}$-lo $(\sim 10 \%)$ and Tau ${ }^{\text {wt }}$-hi ( 15\%) worms at day 1 . All of these fractions increased substantially with time, with $\mathrm{Tau}^{\mathrm{AT}}$-lo animals showing the most advanced mislocalization of mCherry::RAB-3 puncta (see Additional file 12 for an example).

We sought to clarify whether the mislocalization of mCherry::RAB-3 is due to a disruption in the polarized protein trafficking. To this end, PVD sensory neurons in C. elegans represent suitable test cells because they possess distinct axonal-dendritic compartments, with highly branched dendrites encircling the body, while the axon protrudes to the ventral nerve cord and then grows anteriorly $[33,34]$ (see schematic in Fig. 5a). We generated Tau-tg worms carrying kyIs445:Is:[Pdes-2::mCherry::RAB-3;des-2::SAD-1::GFP;odr-1::DsRED] transgene that expresses mCherry fused to RAB-3 in PVD sensory neurons [35]. mCherry::RAB-3 puncta were barely detectable in the PVD dendrites of $\mathrm{Tau}^{\mathrm{wt}}$ (-lo or -hi) animals, similar to non-tg (kyIs445) worms, with only faint signals detectable in Tau ${ }^{\mathrm{wt}}$-hi at day 1 and increasing with age (Fig. 5b, c). Contrary to this, in Tau ${ }^{\mathrm{AT}}$ worms mCherry::RAB-3 was already mislocalized to the entire PVD dendritic compartment at day 1 (Fig. 5b, panel day1 dendritic). At the same time, the axons of Tau ${ }^{\mathrm{AT}}$ worms showed less dense puncta (Fig. 5b, panel day 1 axonal). Altered synaptic vesicle distribution could mean that the axonal compartments are compromised in $\mathrm{Tau}^{\mathrm{AT}}$ worms, implying that there might be a neurotransmission failure at the synapses.

To confirm this, we turned to a complementary approach by using chemicals aldicarb and levamisole that interfere with normal neurotransmission [36]. Aldicarb causes paralysis due to hypercontraction of muscles by inhibiting acetylcholine esterase. Levamisole has a similar effect as an acetylcholine receptor agonist. Normal worms with intact pre- and post-synaptic compartments are sensitive to both chemicals. Worms with either a pre- or a post-synaptic defect show resistance to aldicarb, whereas only worms with a post-synaptic defect show resistance to levamisole. Tau ${ }^{\text {wt }}$-lo and non-tg (N2) worms were sensitive to aldicarb, indicating a normal release of acetylcholine at synapses. However, Tau ${ }^{\mathrm{wt}}$-hi, Tau ${ }^{\mathrm{AT}}$-lo and $\mathrm{Tau}^{\mathrm{AT}}$ hi showed a mild resistance to aldicarb (Fig. 5d). On the other hand, all the tau-transgenic lines were equally sensitive to levamisole and did not differ from non-tg (Fig. 5e). Thus these experiments argue that the pre-synaptic compartments are compromised in tau-tg worms.

Next we analyzed the distribution of another intracellular organelle, mitochondria. The wyEx2709 [Pitr-1::TOM-20 $\left.{ }^{1-54 a a}:: y f p\right]$ is a transgene that expresses YFP-tagged mitochondria in DA9 motor neurons [37]. 

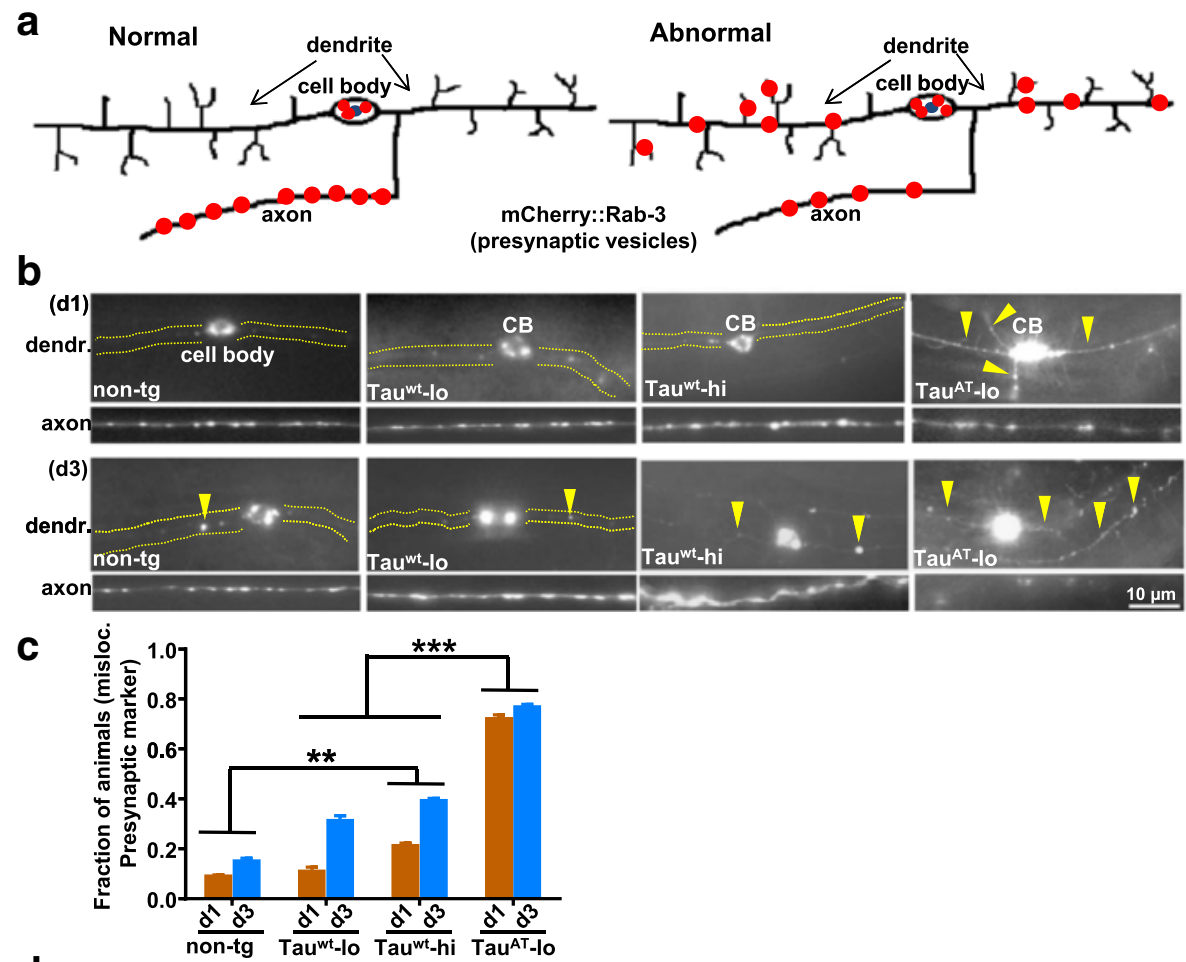

d
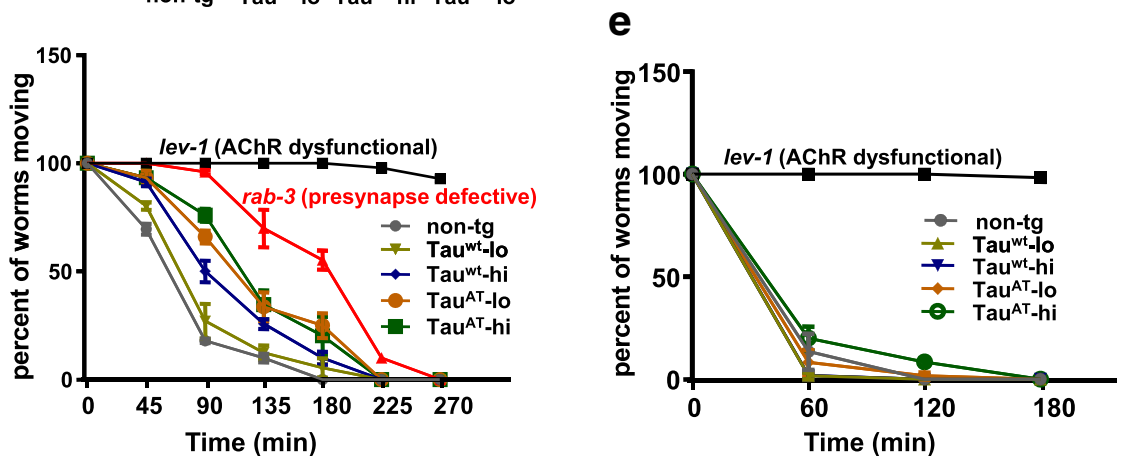

Fig. 5 Mutant Tau ${ }^{A T}$ worms show presynaptic protein mislocalization in dendrites of PVD neurons and neurotransmission defects. a Schematic representation of a PVD neuron with normal localization of mCherry::RAB-3 (pre-synaptic marker, red dots) and a PVD neuron with altered localization. The branched morphology represents the dendritic compartment; the unbranched ventral process is the axon. $\mathbf{b}$ Representative images of dendrites and axons at day 1 and day 3 of the mentioned transgenes. In non-tg and Tau ${ }^{\text {wt }}$ worms (-lo and -hi), mCherry:RAB-3 localizes in the axonal compartment and is excluded from the dendrites in young adults (day 1). By contrast, Tau ${ }^{\mathrm{AT}}-$ lo worms show mCherry::RAB-3 mislocalized to the dendrites (yellow arrowheads) and a reduced distribution in the axons. With age, Tau ${ }^{\text {wt }}$ (-lo and -hi) also show slight mislocalization to the dendritic compartments (yellow arrowheads). Yellow dotted areas correspond to the dendrite not visible in non-tg, Tau ${ }^{\text {wt }-l o}$ and Tau ${ }^{\text {wt }}-$ hi worms at day1. c Quantification of the fractions of animals with mislocalized presynaptic mCherry:RAB-3 puncta in tau-transgenes at day 1 (d1) and day 3 (d3). Non-tg worms serve as control. Error bars denote mean \pm SEM, $n \geq 20$. ${ }^{* *} P \leq 0.01$, ${ }^{* *} P \leq 0.001$. Paired t-test with unequal variance was used for comparison. d Time-dependent paralysis induced by aldicarb (acetylcholine esterase inhibitor). Data represents the percentage of worms (mean \pm SEM) still able to move on $1 \mathrm{mM}$ aldicarb plates after being touched, as a function of time. Non-tg and Tau ${ }^{\text {wt }-l o ~ w o r m s ~ a r e ~ h i g h l y ~}$ sensitive (bottom curves, grey and olive). Tau ${ }^{\text {wt }}$-hi, Tau ${ }^{\mathrm{AT}}$-lo and Tau ${ }^{\mathrm{AT}}$-hi worms show some resistance (blue, ochre, green curves, resp.). Strongly resistant lev-1 (AChR mutant carrying the e211 allele) and mildly resistant rab-3 (Ras GTPase mutant carrying the j549 allele) are used as additional controls (black and red curve, resp). After applying two-way ANOVA with Bonferroni correction, $P<0.01$ at time points 45 and 180 min, whereas $P<0.001$ at time points 90 and 135 min is obtained for Tau ${ }^{\mathrm{Wt}}$-hi, Tau ${ }^{\mathrm{AT}}$-lo and Tau ${ }^{\mathrm{AT}}$-hi against non-tg. $n=20$ animals, three independent repetitions. e Time-dependent paralysis induced by levamisole (acetylcholine receptor agonist). Data represents the percentage of worms (mean \pm SEM) still able to move on $0.2 \mathrm{mM}$ levamisole plates after being touched, as a function of time. All the four tau-lines were as sensitive to levamisole as non-tg. Strongly resistant lev-1 (e211) is shown as an additional control. $n=20$ animals, three independent repetitions 
The DA9 soma lies in the preanal ganglion; it has a well defined dendrite extending anterioventrally and an axon that crosses the body to reach the dorsal cord and then runs anteriodorsally. Non-tg animals display a typical distribution of labelled mitochondria (schematic in Fig. 6a, [37]; such that the anteriodorsal region (boxed area, $\sim 90 \mu \mathrm{m}$, Fig. 6a) contains closely spaced $(\sim 5-10 \mu \mathrm{m})$ mitochondria, as seen in the non-tg reporter strain (wyEx2709) (Fig. 6b, panel non-tg). But away from the anteriodorsal region towards the distal end, the spacing between the mitochondrial particles increases $(\sim 30-50 \mu \mathrm{m})$ (data not shown). We then visualized mitochondria in our tau-tg animals by crossing them into wyEx2709. Tau ${ }^{\mathrm{AT}}$-lo worms presented a smaller number of mitochondria in all the three classified compartments of DA9 neurons compared to non-tg,

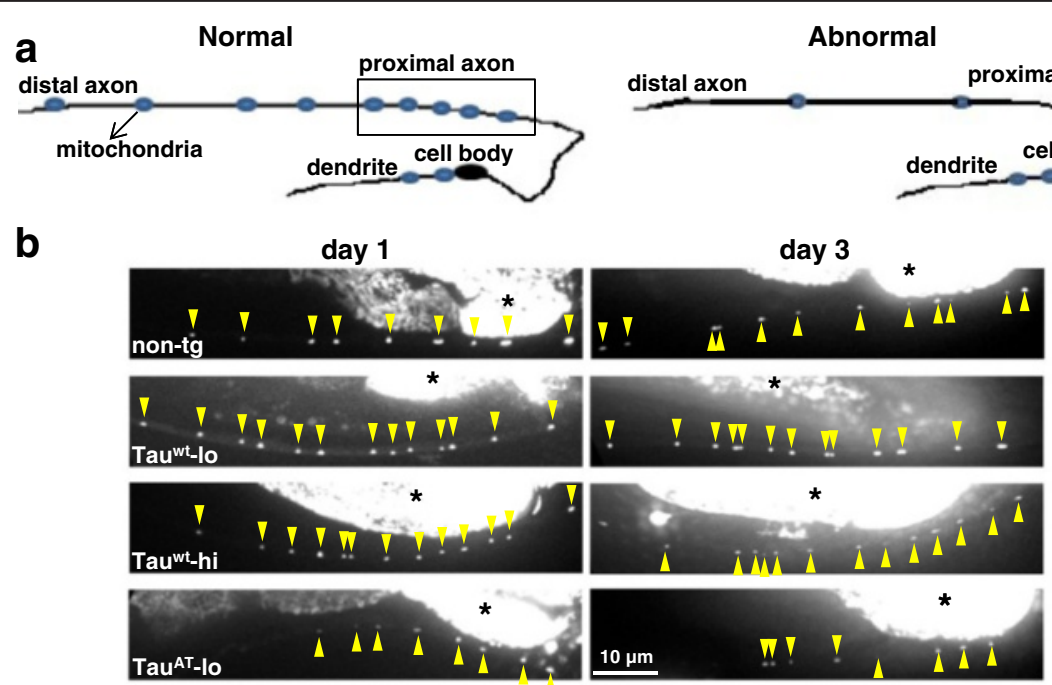

C

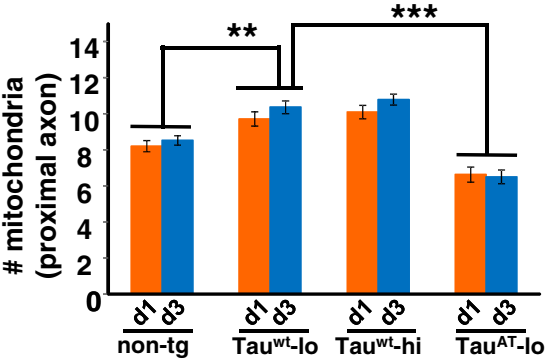

d

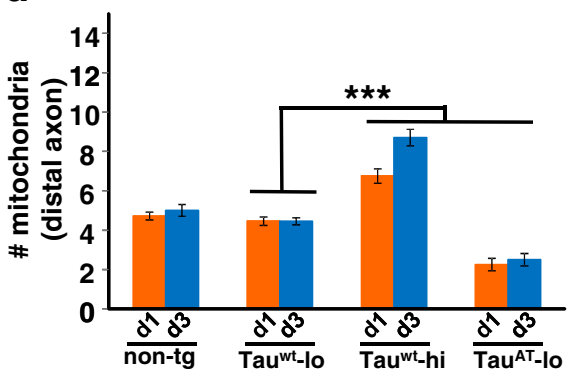

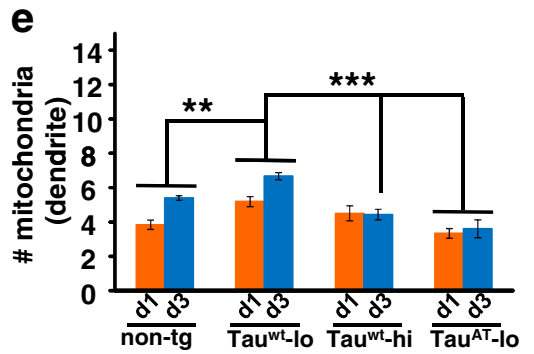

Fig. 6 Mutant Tau ${ }^{A T}$ worms display abnormal distribution of mitochondria. a Schematic representation of DA9 motor neuron displaying a normal and an abnormal patterning of mitochondria in different compartments (blue dots). b Day-1 old adult worms of the tau-transgenes (left panel) visualized after crossing them into the wyEx2709 [Pitr-1::TOM-20 ${ }^{1-54 a a}:$ :yfp] reporter that highlights YFP-tagged mitochondria in DA9 motor neurons. Magnified images of the region corresponding to the proximal axon (boxed area in the schematic) are shown to visualize the faint mitochondrial particles (yellow arrowheads). Mitochondrial particles in day-3 old adult worms of the transgenes (right panel). Areas in white marked by asterisk shows body autofluorescence. $\mathbf{c}, \mathbf{d}$, and $\mathbf{e}$ Quantification of the average number of mitochondria in different regions of DA9 neurons designated as proximal axon (boxed region), distal axon (away from boxed region) and dendrite. Tau ${ }^{\mathrm{AT}}$-lo animals exhibit fewer mitochondria than non-tg and Tau ${ }^{\text {wt }}$ worms in all the specified compartments of the neuron at day 1 and day 3 . Error bars show mean \pm SEM, $n \geq 20 .{ }^{* *} P \leq 0.01$, ${ }^{* * *} P \leq 0.001$. Paired t-test with unequal variance was used for comparison 
$\mathrm{Tau}^{\mathrm{wt}}$-lo and $\mathrm{Tau}^{\mathrm{wt}}$-hi at both the time points (day 1 and day 3) (Fig. 6b). However, compared to non-tg animals, $\mathrm{Tau}^{\mathrm{wt}}$ expression led to an increase in mitochondria in a dose dependent manner. This effect was more pronounced in the distal axon, where $\mathrm{Tau}^{\mathrm{wt}}$-hi worms showed $\sim 1.7$ fold increase in mitochondrial number compared to Tau ${ }^{\text {wt }}$-lo (Fig. 6c, d, e). Collectively, these results show that the mutant $\mathrm{Tau}^{\mathrm{AT}}$ distorts the normal distribution of neuronal cargoes in C. elegans neurons.

\section{Altered mitochondrial distribution in mutant $\mathrm{Tau}^{\mathrm{AT}}$ worms is due to a defective transport}

Altered steady state distribution of major neuronal cargoes (synaptic vesicles and mitochondria) in $\mathrm{Tau}^{\mathrm{AT}}$ animals is likely due to impaired neuronal transport machinery. To investigate the effect of $\mathrm{Tau}^{\mathrm{AT}}$, we performed mitochondrial trafficking analysis by generating Tau-tg worms carrying jsIs609 transgene [17] which expresses GFP fused to a mitochondrial localization signal (MLS) in mechanosensory neurons. In order to get an insight into how the transport machinery is affected in different parts of neurons, we chose two regions, one close to the cell body (up to $\sim 80 \mu \mathrm{m}$ from the cell body) and another in the mid region $(120 \mu \mathrm{m}$ away from the cell body) (refer to Fig. 4a), and recorded mitochondrial movements in a time frame of $2.5 \mathrm{~min}$. The transport behavior was quite different in $\mathrm{Tau}^{\mathrm{AT}}$-lo compared to non-tg (jsIs609) and $\mathrm{Tau}^{\mathrm{wt}}$ animals. Non-tg and Tau ${ }^{\mathrm{wt}}$ animals generally showed long-haul uninterrupted movements (range $10-15 \mu \mathrm{m}$ ), whereas $\mathrm{Tau}^{\mathrm{AT}}$-lo animals displayed short bidirectional movements $(<5 \mu \mathrm{m})$ (Fig. 7a, also see Additional file 5: Movie S5, Additional file 6: Movie S6, Additional file 7: Movie S7 and Additional file 8: Movie S8). Quantification revealed a drastic decrease in the number of events ( 30 to $60 \%$ reduction) in $\mathrm{Tau}^{\mathrm{AT}}$-lo animals compared to non-tg and $\mathrm{Tau}^{\mathrm{wt}}$ (-lo and -hi) animals at both time points. With age, non-tg and $\mathrm{Tau}^{\mathrm{wt}}$-lo worms showed a decrease in the number of trafficking events, especially in the proximal region $\left(\sim 30-35 \%\right.$ reduction), whereas $\mathrm{Tau}^{\mathrm{wt}}-\mathrm{hi}$ and $\mathrm{Tau}^{\mathrm{AT}}$-lo did not display a drastic change in the trafficking events in either of the regions with age (Fig. 7b, c). In conclusion, $\mathrm{Tau}^{\mathrm{AT}}$-lo worms showed a severe defect in mitochondrial transport compared to non-tg and Tau ${ }^{\text {wt }}$ animals, which provides a plausible explanation for the altered steady state distribution of neuronal cargoes in the worms expressing mutant $\mathrm{Tau}^{\mathrm{AT}}$.

\section{Mutant $\mathrm{Tau}^{\mathrm{AT}}$ adopts a pathological conformation but does not form insoluble aggregates}

A common feature of neurodegenerative diseases is protein aggregation. There is an ongoing debate regarding the nature of the toxic species, e.g. the relatively stable insoluble aggregates which could obstruct the cellular space, or soluble aggregates in the form of oligomers [38-40]. To address this question for the case of the $\mathrm{Tau}^{\mathrm{AT}}$ mutant, we first biochemically extracted tau from day-1 old worms [17]. Soluble fractions were completely extracted first by using a salt buffer (RAB), which removes most of the soluble cytoplasmic fraction, followed

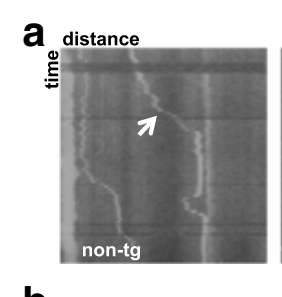

b

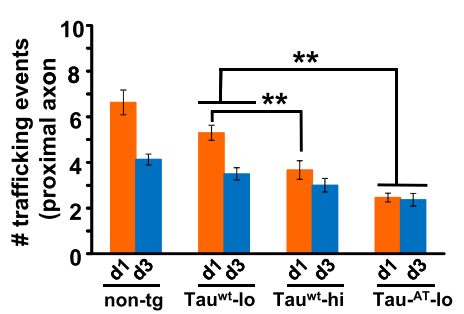

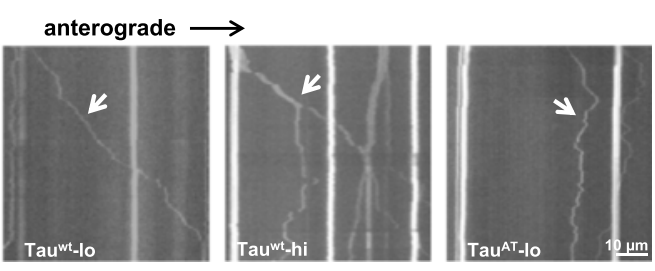

C

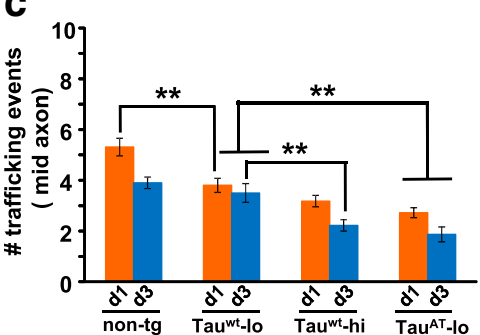

Fig. 7 Time-resolved imaging of mitochondria reveals deficits in the trafficking machinery of mutant Tau ${ }^{A T}$ animals. a Representative kymographs showing the nature of particle movements in different worm lines. Mitochondria in non-tg, Tau ${ }^{\mathrm{wt}}$-lo and Tau ${ }^{\mathrm{wt}}$-hi worms show long range movements (in the range 8-15 $\mu \mathrm{m}$ ) compared to Tau ${ }^{\mathrm{AT}}$-lo where mitochondria exhibit short and oscillatory movements $(<5 \mu \mathrm{m})$. Quantitation of number of mitochondrial trafficking events in proximal (b) and mid region (c) of mechanosensory neuron in day 1 and day 3 adults. Tau ${ }^{\text {AT-lo animals }}$ show significant reduction in mitochondrial trafficking compared to non-tg and Tau ${ }^{\text {wt }}$-lo both at day 1 and day 3 . However, Tau ${ }^{\text {wt }}$ showed dose dependent decreasing trends of trafficking events in both the regions of the neuron. Error bars show mean \pm SEM, $n \geq 10$. ${ }^{* *} P \leq 0.001$. Paired t-test with unequal variance was used for comparison 
by a detergent buffer (RIPA), which removes proteinprotein or protein-membrane complexes. The detergent insoluble pellet was finally reextracted with urea buffer (Urea). Neither $\mathrm{Tau}^{\mathrm{wt}}$ nor $\mathrm{Tau}^{\mathrm{AT}}$ accumulated detergent insoluble tau at day-1 (Additional file 13). Next we extracted tau from mixed stage worms consisting mostly of $>3$ days old ones. Here we used CK10 line which is transgenic for htau ${ }^{\mathrm{V} 337 \mathrm{M}}$ (1N4R) and is known to accumulate detergent insoluble tau with age (mixed stage) as a positive control [41]. The CK10 line but not $\mathrm{Tau}^{\mathrm{wt}}$ or $\mathrm{Tau}^{\mathrm{AT}}$ showed tau in the detergent insoluble fraction (Fig. 8a), although there was an increase in the detergent soluble Tau fraction in $\mathrm{Tau}^{\mathrm{wt}}$ and $\mathrm{Tau}^{\mathrm{AT}}$ with age compared to the day- 1 old adults (see Additional file 13 for comparison).

To confirm our results, we generated another line expressing a Tau chimaera with two additional proline substitutions in the hexapeptide motifs (I277P and I308P), besides the A152T mutation (termed Tau ${ }^{\mathrm{AT}+\mathrm{PP}}$ ) (Fig. 8b). The proline substitutions make this Tau chimaera inherently incapable of forming detergent insoluble Tau filaments because they block the amyloidogenic $\beta$-structure [42]. We then compared the protein levels in the worms carrying $\mathrm{Tau}^{\mathrm{wt}}, \mathrm{Tau}^{\mathrm{AT}}$ or $\mathrm{Tau}^{\mathrm{AT}+\mathrm{PP}}$ transgenes as extrachromosomal arrays at day 1 , all of which showed comparable levels of Tau protein (Fig. 8c). Contrary to

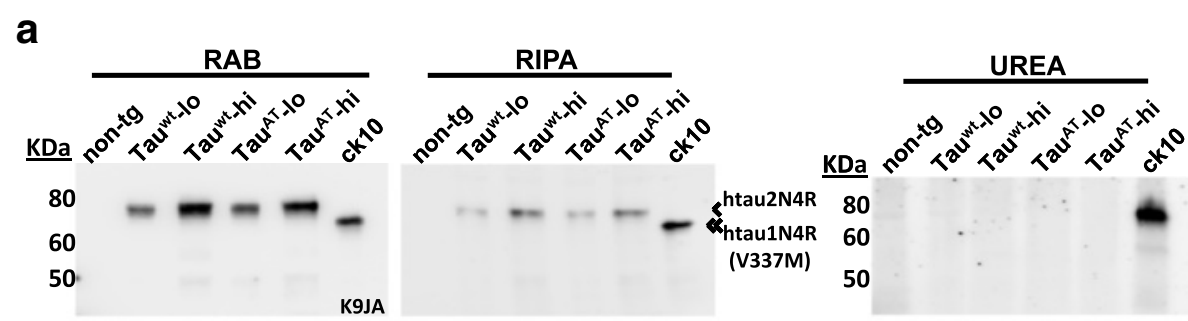

tubulin ... ........
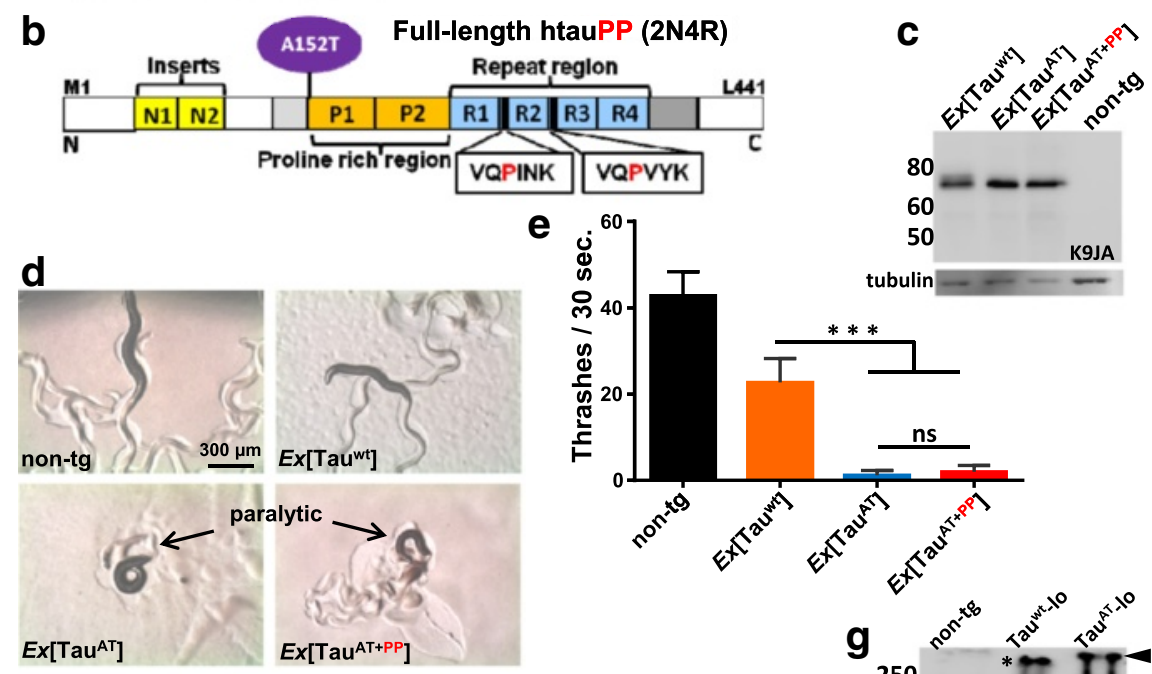
expectations, $\mathrm{Tau}^{\mathrm{AT}+\mathrm{PP}}$ resulted to be equally toxic and the $\mathrm{Tau}^{\mathrm{AT}+\mathrm{PP}}$ worms were as paralytic as single mutant $\mathrm{Tau}^{\mathrm{AT}}$ worms (Fig. 8d,e). This argues that the toxicity does not depend on the formation of $\beta$-structure and incipient aggregation, in contrast to the pro-aggregant mutation $\Delta \mathrm{K} 280$ described earlier $[17,43]$.

Before the accumulation of insoluble aggregates as tangles, Tau is known to undergo a change towards a pathological conformation which accompanies oligomerization during the pre-tangle state and can be recognized by antibodies Alz-50 or MC-1 [44]. Using MC-1 staining, we confirmed pathological conformation and observed an extensive staining of nerve ring ganglion and nerve cords in $\mathrm{Tau}^{\mathrm{AT}}$-lo worms. By contrast, $\mathrm{Tau}^{\mathrm{wt}}$ (-lo and -hi) worms showed only minimal staining (Fig. 8f) and non-tg worms showed no staining (data not shown). Since it was reported previously that $\mathrm{Tau}^{\mathrm{AT}} \mathrm{mu}-$ tant tau has a higher tendency to form oligomers in vitro [6], we wanted to test if such oligomeric species also exist in $\mathrm{Tau}^{\mathrm{AT}}$ worms. To detect oligomers, we applied native PAGE to analyze total lysate extracted from the mixed stage worms in buffer $C$ (see Methods) using K9JA antibody. Lysate from $\mathrm{Tau}^{\mathrm{wt}}$-lo showed protein accumulated in two bands $(\sim 170 \mathrm{KDa}$ and $>250 \mathrm{KDa}$ marked by asterisk in Fig. 8g). By contrast, the lysate from $\mathrm{Tau}^{\mathrm{AT}}$-lo showed in addition a higher band (Fig. 8g, black arrowhead) at the top of the gel and a smear in the range of $72-95 \mathrm{KDa}$ (Fig. $8 \mathrm{~g}$, line), meaning that $\mathrm{Tau}^{\mathrm{AT}}$ worms accumulate a broad range of higher molecular weight tau species compared to $\mathrm{Tau}^{\mathrm{wt}}$ worms. Lysate from non-tg worms on the other hand did not show any reaction to K9JA antibody. We conclude that the mutation A152T favors a pathological conformation which might promote oligomerization.

\section{The toxicity of mutant $\mathrm{Tau}^{\mathrm{AT}}$ is not prevented by anti- aggregation compounds}

The conclusion that the toxicity of A152T mutation occurred independently of the $\beta$-propensity of the repeat domain was corroborated by treatment of $\mathrm{Tau}^{\mathrm{AT}}-\mathrm{lo}$ worms with inhibitors of Tau aggregation (Rhodanine compound bb14 and PTH compound BSc3094), that were competent to ameliorate the phenotype of a worm model based on tau aggregation [17]. The $\mathrm{Tau}^{\mathrm{AT}}$-lo worms were treated with varying concentrations of compounds in liquid culture for three days, starting from the L1 larval stage. However, the compounds failed to improve the paralytic phenotype of the $\mathrm{Tau}^{\mathrm{AT}}$-lo worms (Fig. 8h). This implies that the principle behind the pathological features observed in $\mathrm{Tau}^{\mathrm{AT}}$ worms is perhaps different from that of the conventional Tau-aggregation.

\section{The C-terminal half of Tau including the repeat domain is necessary for the toxicity of human tau in C. elegans neurons}

The repeat domain of tau in the C-terminal domain harbors the primary physiological and pathological functions of tau, stabilization of microtubules and formation of aggregates in disease [45, 46]. Much less is known about the functions of the N-terminal domain; but it is known to interact with motor proteins and signalling proteins [9, 47-49]. Additionally, the pathological conformation adopted by tau is achieved as a result of intramolecular folding of $\mathrm{C}$-terminal against the $\mathrm{N}$-terminal

\section{(See figure on previous page.)}

Fig. 8 Mutant Tau ${ }^{A T}$ does not aggregate, adopts a pathological conformation and anti-aggregation compounds do not rescue the paralysis. a Sequential extraction of tau from aged animals (mixed stage consisting worms of mostly $>3$ days old). CK10 line transgenic for htauV337M (1N4R) accumulates detergent insoluble tau (insoluble aggregated tau); while no insoluble tau is detected in Tau ${ }^{\text {wt }}$ or Tau ${ }^{\text {AT }}$ lines. 2N4R-tau (single arrow) in Tau ${ }^{\text {wt }}$ and Tau ${ }^{\text {AT }}$ lines migrates slower than the 1N4R-tau (double arrow) in CK10 worms. With age, more Tau accumulates in the detergent soluble fraction (representing Tau bound to membranous structures) (see also Additional file 13A for day-1 old animals). b Bar diagram of anti-aggregant Tau construct Tau ${ }^{\text {AT+PP }}$ with

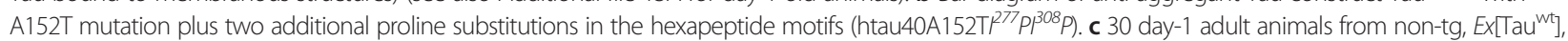
Ex[Tau $\left.{ }^{\mathrm{AT}}\right]$ and its anti-aggregant variant Ex[Tau $\left.{ }^{\mathrm{AT}+\mathrm{PP}}\right]$ after lysis in $1 \times$ sample buffer, then subjected to $10 \%$ PAGE and subsequent western blot analysis using K9JA-pan-tau antibody. Ex[Tau $\left.{ }^{\mathrm{Wt}}\right]$, Ex[Tau $\left.{ }^{\mathrm{AT}}\right]$ and its anti-aggregant variant Ex[Tau $\left.{ }^{\mathrm{AT}+\mathrm{PP}}\right]$ carrying the respective Tau transgenes as extrachromosomal arrays (Ex) show comparable levels of Tau expression. Tubulin serves as loading control. $\mathbf{d}$ Micrographs of the worms. The anti-aggregant variant of mutant Tau $^{\text {AT }}\left(\right.$ Tau $^{\text {AT+PP }}$ ) is equally toxic and produces a similar paralytic phenotype as the single mutant Tau ${ }^{\text {AT }}$. Note the absence of tracks and coiled body in Ex $\left[T_{a u^{A T+P P}}\right]$ similar to Ex[Tau $\left.{ }^{A T}\right]$. e Mean thrashing assay of day-1 old adult animals carrying Tau ${ }^{\text {wt }}$, Tau ${ }^{\text {AT }}$ or its anti-aggregant variant (Tau ${ }^{\text {AT }+P P}$ ) transgenes as extrachromosomal arrays. Ex[Tau ${ }^{\mathrm{AT}+\mathrm{PP}}$ ] shows less thrashes than the non-tg ( $5 \%$ of non-tg) or Ex[Tau $\left.{ }^{\text {wt }}\right]$ worms ( 9 \% of Tau ${ }^{\text {wt }}$ worms). But there is no difference between the Ex[Tau $\left.{ }^{\mathrm{AT}}\right]$ and its anti-aggregant variant Ex $\left[\operatorname{Tau}^{\mathrm{AT}+\mathrm{PP}}\right]$, indicating that the toxicity does not depend on amyloidogenic aggregation. Non-tg strain serves as control. Error bars denote SEM, $n \geq 30$. ${ }^{* *} P<0.001$, ns., not significant. One-way ANOVA with Tukey's test applied for multiple comparisons. $\mathbf{f}$ Immunostaining of day-1 old Tau ${ }^{\text {wt }}$-lo, Tau ${ }^{\text {wt }}$-hi and Tau ${ }^{\text {AT }}$-lo with conformation-specific antibody MC1. Tau in Tau ${ }^{\text {AT }}$-lo worms adopts a pathological state as seen by dense staining in the nerve ring and ventral cord. White arrows show stained neuronal processes either in the nerve ring or ventral cord region. Tau wt (-lo and -hi) show only mild staining occasionally. $\mathbf{g}$ Worm extracts prepared from mixed stage adults in buffer $\mathrm{C}$, resolved by native PAGE and immunoblotted with K9JA show Tau enriched in soluble high molecular weight complexes in Tau ${ }^{\text {AT }}$ worm extracts. In addition to two bands common to both Tau ${ }^{\text {wt }}$-lo and Tau ${ }^{\mathrm{AT}}$-lo lysates ( $170 \mathrm{KDa}$ and $>250 \mathrm{KDa}$ marked by asterisks), a smear in the range of $72-95 \mathrm{KDa}$ corresponding to lower oligomeric species and a higher band ( $>>250 \mathrm{KDa}$, black arrowhead) can be seen in the Tau ${ }^{\mathrm{AT}}$-lo lysate. $\mathbf{h}$ Mean number of bends per $30 \mathrm{~s}$ of Tau $^{\text {AT }}$-lo worms treated either with DMSO (solvent control) or with 50 or $100 \mu$ M concentrations each of known aggregation inhibitors of Tau (Rhodanine compound bb14, PTH compound BSc3094 in DMSO). The lack of rescue indicates that the toxicity of Tau ${ }^{\text {AT }}$ is based on some mechanism distinct from aggregation (for comparison see [17]). Error bars denote SEM. One-way ANOVA with Tukey's test applied for multiple comparisons (ns., not significant) 
domains (discontiguous epitope MC-1, residues 5-15+ 312-322) [44]. Furthermore, mimicking the posttranslational modifications at epitopes of antibodies AT8, AT100 and PHF-1 (upstream and downstream of the repeats, resp.) leads to a change of the paperclip folding of Tau that is known to consolidate the MC-1 epitope [50]. Since the A152T mutation lies in the Nterminal projection domain of tau, we wondered if $\mathrm{N}$ terminal tau domains that lack a potential MC-1 epitope would still produce a similar phenotype as the fulllength tau. We generated transgenic worms expressing pan-neuronally the $\mathrm{N}$-terminal tau (Nt-tau) chimaeras $\left(\mathrm{Met}^{1}{ }^{-} \mathrm{Leu}^{243}\right.$ ) with or without the mutation A152T (Fig. 9a). Several lines carrying the N-terminal tau transgenes with or without the mutation $\left(E x\left[\mathrm{Tau}^{\mathrm{AT}-\mathrm{Nt}}\right]\right.$ and $E x\left[\mathrm{Tau}^{\mathrm{wt}-\mathrm{Nt}}\right]$ respectively) as extrachromosomal arrays were obtained by expressing the respective transgenes at comparable levels. However, compared to worms expressing full-length htau40 ( $\mathrm{Tau}^{\mathrm{wt}}$ or $\left.\mathrm{Tau}^{\mathrm{AT}}\right)$ as extrachromosomal arrays $\left(E x\left[\mathrm{Tau}^{\mathrm{wt}}\right]\right.$ and $E x\left[\mathrm{Tau}^{\mathrm{AT}}\right]$ respectively), worms expressing N-terminal fragments $\left(E x\left[\mathrm{Tau}^{\mathrm{wt}-\mathrm{Nt}}\right]\right.$ and $\left.E x\left[\mathrm{Tau}^{\mathrm{AT}-\mathrm{Nt}}\right]\right)$ showed much higher expression (5-10 fold) (Fig. 9b). In spite of these high levels, the N-terminal tau fragments produced only a slight reduction in mobility (thrashes) compared to nontg at day 1 and day 5 , and the difference became statistically insignificant at day 7 (Fig. 9c). These results are consistent with earlier findings where an N-terminal fragment of Tau expressed in mouse brain produced phenotypically normal mice [48].

We then performed staining of worms expressing the $\mathrm{N}$-terminal tau fragments with the polyclonal antibody SA4473 specific for the N-terminal half of tau. Nt-tau fragments in these worms showed extensive staining exclusively in somata and nuclei, but were largely excluded from the neuronal processes (Fig. 9d). These results
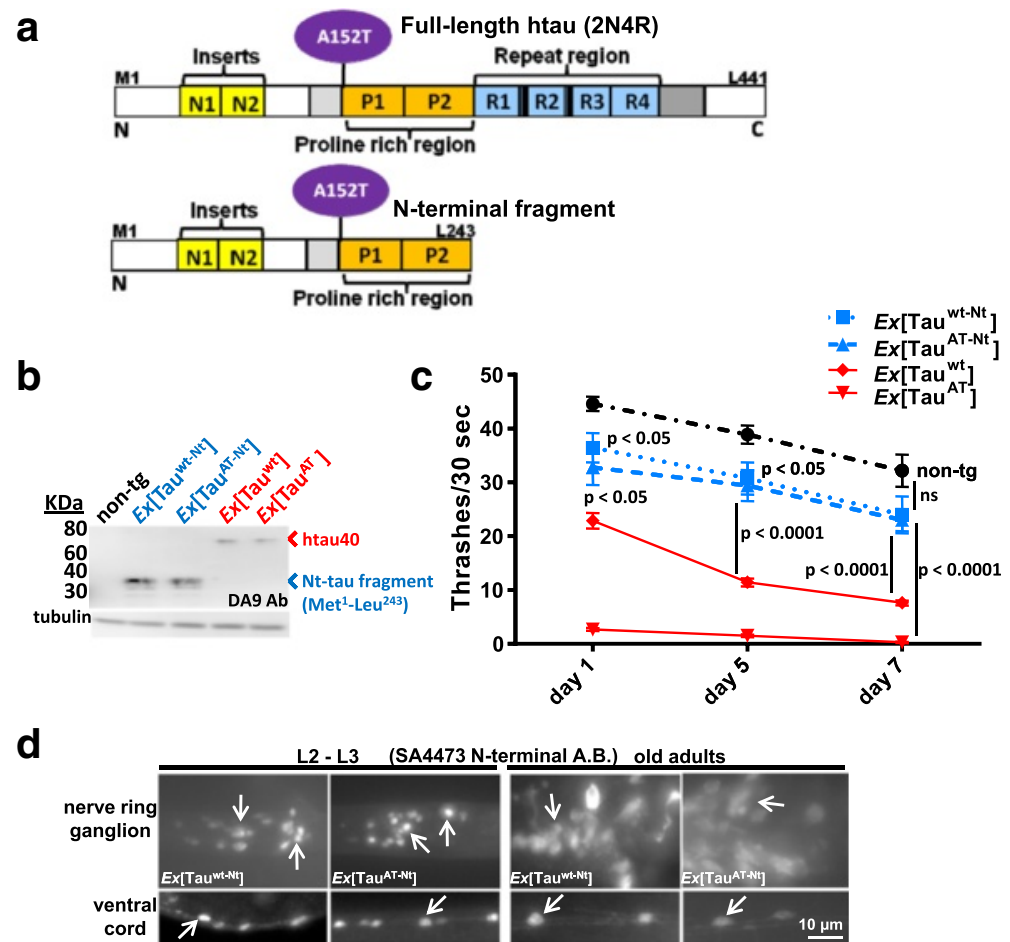

Fig. $9 \mathrm{~N}$-terminal tau fragments (wild-type or with A152T mutation) are not toxic to C. elegans neurons. a Bar diagram depicts the N-terminal fragment of tau (amino acids Met'-Leu ${ }^{243}$ ) derived from full-length Tau. The pan-neuronal snb-1 promoter drives the expression of wild-type $\mathrm{N}$-t- or mutant N-t-fragment (Tau ${ }^{\mathrm{wt}-\mathrm{Nt}}$ and Tau ${ }^{\mathrm{AT}-\mathrm{Nt}}$ respectively) in C. elegans neurons. b Blot showing the protein expression levels in worms carrying $\mathrm{N}$-terminal fragments or full-length tau transgenes as extrachromosomal arrays. 30 synchronized day-1 old adult worms from each

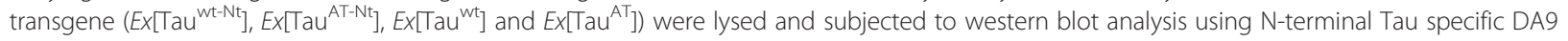
antibody (epitope at aa 100-130). Non-tg worms serve as control and tubulin as loading control. c Age-related comparison of rate of body thrashing in liquid for days 1, 5 and 7 from the respective transgenic animals. In contrast to full-length tau (Tau ${ }^{\text {wt }}$ or Tau ${ }^{\mathrm{AT}}$ ), expression of $\mathrm{N}$-terminal Tau fragments in C. elegans neurons (Tau ${ }^{\mathrm{wt}-\mathrm{Nt}}$ or Tau ${ }^{\mathrm{AT}-\mathrm{Nt}}$ ) cause only slight reductions $(\sim 10-13 \%)$ in thrashing at day 1 and day 5 . The data points represent the mean $( \pm$ SEM) thrashing rate and time point, $n \geq 30$. Two-way ANOVA followed by Bonferroni correction was used for multiple comparisons. $\mathbf{d}$ Immunostaining of animals expressing either wild-type- or mutant- $\mathrm{N}$-t tau fragments at different time points with $\mathrm{N}$-terminal tau-specific antibody SA4473. Top panels depict nerve ring ganglion while the bottom panels show nerve cords. $\mathrm{N}$-terminal Tau fragments show localization restricted to cell bodies and nuclei (white arrows), as nerve processes are largely invisible, in contrast to the situation with full-length Tau (see Fig. 1c) 
show that $\mathrm{N}$-terminal tau fragments which are unable to move into the neuronal processes are not toxic to C. elegans neurons. Thus a mild uncoordinated phenotype due to $\mathrm{Tau}^{\mathrm{wt}}$ and a strong paralytic phenotype due to $\mathrm{Tau}^{\mathrm{AT}}$ could both be abolished by preventing the Tau chimaeras to move into the neuronal processes. In conclusion, a severe toxicity occurs only when both conditions are met; (a) presence in neuronal processes, (b) A152T mutation. Therefore, we speculate that it might be the ability of full-length tau to move into different neuronal processes owing to its C-terminal half that makes it toxic and the A152T mutation further aggravates the problem, whereas restricting its localization to the cell body by abolishing the $\mathrm{C}$-terminal half renders it non-toxic, even in the mutant form. Table 3 sums up the data on the effect of various Tau variants when expressed pan-neuronally in C. elegans.

\section{Discussion}

In this study we used a C.elegans model to examine the toxicity of a rare mutation in the MAPT gene (A152TMAPT) that was recently discovered as a risk factor for frontotemporal dementia spectrum disorders (FTD, PSP, CBD, AD, and others) [51]. Considering that most Tau mutations lie in or near the repeat domain and thus affect Tau-microtubule binding and Tau aggregation, the A152T mutation is unusual in that it lies in the proline-rich region far upstream of the repeat domain $[7,8]$, with only weak effects on Tau aggregation [6]. This poses the question of an alternative mechanism of toxicity. Over the years, several functions have been assigned to the $\mathrm{N}$-terminal domain of tau which do not coincide with the functions of the repeat domain [9]. Examples of interactors with the $\mathrm{N}$-terminal domain are p150/Dynactin $[47,52]$, or signalling kinases containing SH3 domains $[48,49,53]$, which point to functions distinct from microtubule stabilization.

We found that elevation of wild-type human Tau $\left(\mathrm{Tau}^{\mathrm{wt}}\right)$ in C. elegans neurons is already mildly toxic and can induce late-onset pathology in a dose-dependent manner, consistent with results from mouse models of
$\mathrm{AD}$ [54]. However, mutant $\mathrm{Tau}^{\mathrm{AT}}$ at similar levels induces a much more pronounced pathology with an early onset. These worms developed severe motor dysfunction, paralysis and neurodegeneration of GABAergic neuronal processes (Figs. 1 and 2). Since we observed neurodegeneration in motor neurons, a condition often associated with old age in humans [55], we hypothesized that $\mathrm{Tau}^{\mathrm{AT}}$ could cause effects similar to those observed in aging neurons. This can be tested on mechanosensory neurons by well-defined parameters [24], and indeed the neurons of $\mathrm{Tau}^{\mathrm{AT}}$ worms showed early signs of morphological abnormalities such as bendings and extra outgrowths from neuronal processes and somata (Fig. 3b, c) compared to non-tg and Tau ${ }^{\mathrm{wt}}$ worms. The severity of these morphological abnormalities increased with age (Fig. 3c). Moreover, these signs of early aging on the level of $\mathrm{Tau}^{\mathrm{AT}}$-expressing neurons corresponded to a drastic reduction in life-span of the animals as well (Fig. 3e). Since mutations in genes that reduce the lifespan also cause early onset of age-dependent morphological abnormalities in neurons $[23,24,56]$, it is safe to speculate that the pathways that are involved in inducing such age-dependent morphological abnormalities might get activated early in Tau ${ }^{\mathrm{AT}}$ worms. However, some alternate pathways that induce similar morphological changes in neurons independently of aging have also been reported [23, 34].

What could be the reasons behind the neuronal dysfunction and age-related phenotypes in $\mathrm{Tau}^{\mathrm{AT}}$ worms? Tau interacts with microtubules, microtubules are the tracks for intracellular transport, and defects in neuronal transport machinery have been linked to neurodegeneration. This led us to search for signs of transport defects in the neurons of $\mathrm{Tau}^{\mathrm{AT}}$ worms. This was done for the cases of two major neuronal cargoes, namely synaptic vesicle (carrying Rab-3 GTPase) and mitochondria [57]. The $\mathrm{Tau}^{\mathrm{AT}}$ worms presented a perturbed distribution of both of these cargoes. For example, mCherry::RAB-3 puncta accumulated in distal axons and in cell bodies but were depleted in the mid region of mechanosensory neurons (Fig. 4, Additional file 12), consistent with

Table 3 Summary of different Tau variant transformations in C. elegans neurons (Toxicity index: \%age reduction in thrashes when normalized to non-tg)

\begin{tabular}{|c|c|c|c|}
\hline Strain & Sub-cellular localization & Phenotype (Unc) & Toxicity index \\
\hline non-tg & & Non & 0 \\
\hline Full-length (2N4R) hTau40 wt-lo & Cell bodies and neuronal processes excluding nucleus & Weak & $\sim 30$ \\
\hline Full-length (2N4R) hTau40 ${ }^{\text {wt }}$-hi & Cell bodies and neuronal processes excluding nucleus & Mild & $\sim 60$ \\
\hline Full-length (2N4R) hTau40AT (lo/hi) & Cell bodies and neuronal processes excluding nucleus & Strong & $\sim 90$ \\
\hline Full-length (2N4R) hTau40 AT+PP & Cell bodies and neuronal processes excluding nucleus & Strong & $\sim 90$ \\
\hline N-terminal hTau' ${ }^{\text {wt }}\left(\right.$ Met $^{1}$-Leu $\left.{ }^{243}\right)$ & Cell bodies and neucleus excluding neuronal processes & No obvious phenotype & $\sim 13$ \\
\hline N-terminal hTau ${ }^{\mathrm{AT}}\left(\right.$ Met $^{1}$-Leu $\left.{ }^{243}\right)$ & Cell bodies and neucleus excluding neuronal processes & No obvious phenotype & $\sim 15$ \\
\hline
\end{tabular}

Transgenic lines based on different Tau constructs are compared to the non-transgenic control (N2) animals 
defective retrograde and anterograde transport [58-60]. Moreover, not only the transport but the integrity of the neuronal compartments was compromised in mutant $\mathrm{Tau}^{\mathrm{AT}}$ worms as suggested by the mislocalization of mCherry::RAB-3 in the dendritic compartments of polarized PVD neurons (Fig. 5b, c) [33, 35].

Polarized sorting of synaptic vesicles requires ankyrin (UNC-44) driven anchoring of CRMP (UNC-33) into axons, which in turn drives plus-end directed microtubules extending into the axons and subsequent plus-end directed axonal traffic by kinesin (UNC-104) [35]. The observed missorting of synaptic vesicle associated RAB-3 into dendrites of $\mathrm{Tau}^{\mathrm{AT}}$ worms could be caused by several factors, e.g. more plus-end directed microtubules in dendrites, or mislocalization of ankyrin (UNC-44) and subsequent localization of CRMP (UNC-33) into dendrites, both of which are normally excluded from this compartment. The resulting changes in the dendritic microtubular network due to CRMP could then trigger kinesin (UNC104) dependent traffic into the dendrites. Future studies should reveal if this phenomenon in $\mathrm{Tau}^{\mathrm{AT}}$ worms is related to a direct interaction of tau with ankyrin (UNC-44) or CRMP (UNC-33). In addition to the microtubular network, tau is also known to interact and directly or indirectly influence the dynamics of the actin cytoskeleton [61], and this cross-talk with the microtubule- and actincytoskeleton might play a critical role in tau-induced neurodegeneration [62]. In line with these findings, two suppressors (sut-1 and sut-2) have been identified in a genetic screen using C. elegans $[15,16]$. Although a direct interaction between tau and SUT-1 or SUT-2 is not known; however, both of these proteins interact with proteins associated with either microtubule-based or actin-based cytoskeletal systems.

A similar correlation was seen with mitochondrial particles tagged with YFP in DA9 motor neurons. Mutant $\mathrm{Tau}^{\mathrm{AT}}$ worms showed less mitochondrial particles in the synaptic areas of DA9 axons. Time-resolved imaging revealed that this perturbed mitochondrial distribution is due to a decreased mitochondrial trafficking in $\mathrm{Tau}^{\mathrm{AT}}$ worms (Figs. 6 and 7). This perturbed distribution of neuronal organelles is analogous to the previous C. elegans kinesin/dynein double mutant studies which reported an overall decrease in the number density of synaptic vesicles along the axonal processes and accumulation of very few vesicles in the end neurons. This can be explained by the fact that both retrograde and anterograde transport processes are affected in a kinesin/dynein double mutant. On the other hand, the expression of $\mathrm{Tau}^{\mathrm{wt}}$ generated a phenotype analogous to a dynein mutant where a deficient retrograde transport leads to the accumulation of synaptic vesicles along the distal part of the axon $[59,60]$. These transport deficits might also explain the extensive neuronal restructuring of the touch neurons in Tau ${ }^{\mathrm{AT}}$ animals (Fig. 3). The fact that most of these non-specific branches grew directly from the cell bodies supports the view that mitochondria accumulate in the cell bodies which may provide local energy for new outgrowths. Occasionally these nonspecific branches in touch neurons of $\mathrm{Tau}^{\mathrm{AT}}$ worms underwent further branching, consistent with earlier studies that attributed this phenomenon to ROS production by static dysfunctional mitochondria [24]. Overtime, even wild-type $\mathrm{Tau}^{\mathrm{wt}}$ worms started showing these phenotypes, albeit to a lesser extent.

It is worth noting that in certain assays, $\mathrm{Tau}^{\mathrm{wt}}$ and $\mathrm{Tau}^{\mathrm{AT}}$ worms did not differ much, possibly because a threshold in these phenotypic assays is lower and even the wild-type tau was able to cross that threshold, albeit at higher levels. For instance, Tau ${ }^{\mathrm{wt}}$ at higher levels led to pre-synaptic defects similar to $\mathrm{Tau}^{\mathrm{AT}}$ at both lower and higher levels (Fig. 5d, e). Similarly, there was no change in the phosphorylation status of tau in both $\mathrm{Tau}^{\mathrm{wt}}$ and $\mathrm{Tau}^{\mathrm{AT}}$ worms (Additional file 14). This indicates that there is no special preference for mutant tau with regard to phosphorylation by C. elegans kinases and further suggests that tau phosphorylation may not be a cause for toxicity. In addition, neither Tau ${ }^{\text {wt }}$ nor $\mathrm{Tau}^{\mathrm{AT}}$ worms accumulated insoluble tau (Fig. 8a).

Aggregation is usually considered a hallmark of Tauopathies such as AD, PSP, or CBD, but there is also persuasive evidence that the toxic species are not the fully developed insoluble and aggregated forms of the protein but earlier soluble oligomeric precursors [63-65]. We therefore searched for assembly forms of $\mathrm{Tau}^{\mathrm{AT}}$ in the transgenic worms. The results showed that there are no detectable insoluble aggregates (as judged by the usual standard isolation procedures), but an increase in soluble oligomeric forms. In this regard, the Tau ${ }^{\mathrm{AT}}$ are distinct from other cases where aggregation-prone Tau mutants were expressed and accumulated in the insoluble fraction in C. elegans neurons, e.g. mutants V337M or $\Delta \mathrm{K} 280$ [17, 41]. The absence of insoluble aggregates was further confirmed in two ways: (i) by expressing an inherently aggregation-incompetent Tau construct with two proline substitutions in the hexapeptide motifs plus the A152T mutation which yielded a similar phenotype as a tau construct with the A152T mutation alone (Fig. $8 \mathrm{~d}, \mathrm{e}$ ); and (ii) by treating the $\mathrm{Tau}^{\mathrm{AT}}$ worms with known aggregation inhibitor compounds, which offered no relief (Fig. 8h), in contrast to worms expressing pro-aggregant forms of Tau [17]. These experiments argue that the toxic principle of $\mathrm{Tau}^{\mathrm{AT}}$ does not depend on aggregation. Important to mention here is that another line expressing a full-length htau40A152T chimaera with an additional V337M mutation $\left(E x\left[\mathrm{Tau}^{\mathrm{AT}+\mathrm{VM}}\right]\right)$ also gave a similar phenotype as the $E x\left[\mathrm{Tau}^{\mathrm{AT}}\right]$ line expressing the single mutant tau chimaera htau40A152T (Additional file 13C,D). Keeping in mind 
that the A152T mutation lies in a region important for scaffolding and signalling pathways, it further suggests that the mutation leads to a toxic gain of function distinct from aberrant aggregation. Indeed, the $\mathrm{Tau}^{\mathrm{AT}}$ worms showed strong staining with antibody $\mathrm{MC}-1$ (Fig. 8f), a characteristic feature of pathological conformational changes [44]. Thus the situation is reminiscent of other neurodegenerative conditions where soluble pre-aggregates are the primary culprits behind the toxicity [66-68]. In line with these findings, tau in A152T worms was detected mostly enriched in the soluble higher molecular weight species (potential oligomers) by native PAGE (Fig. 8g). A growing body of evidence suggests that a cell combats the decline of proteostasis due to toxic gain of function of proteins by sequestering soluble proteins into insoluble aggregates [69, 70]. The fact that $\mathrm{Tau}^{\mathrm{AT}}$ worms have a short life-span (Fig. 3e) might explain that there is not enough time to sequester and circumvent toxic effects caused by soluble diffusible tau. It would be of interest to investigate whether an increase of life-span, e.g. by suppressing insulin-signalling [71], would allow them to drive these soluble tau species into insoluble aggregates, and subsequently whether the pathological consequences are alleviated.

One important finding of this study was that the $\mathrm{Tau}^{\mathrm{AT}}$ mutant does not induce pathology unless tau moves into the neuronal processes. It appears that $C$. elegans neurons can easily cope with a tau chimaera, irrespective of whether it is wild-type or a A152T mutant, provided that its localization is restricted to the cell body. The evidence is that when expressing wild-type or A152T mutant $\mathrm{N}$-terminal tau fragments without the repeat domain, their localization was restricted to cell body (Fig. 9d). The explanation is that the fragments cannot engage to the microtubule-based transport machinery since they lack the MT-binding domain [72]. Both these lines were almost as healthy as the non-tg lines (Fig. 9c), even though the $\mathrm{N}$-terminal fragments were expressed at a 5-10 fold higher level than fulllength tau (Fig. 9b). Secondly, a full-length variant of Tau with two additional proline substitutions in the hexapeptide motifs $\left(\mathrm{Tau}^{\mathrm{AT}+\mathrm{PP}}\right.$ ) which is incompetent for aggregation, but otherwise is transported normally into cell processes (Additional file 15), induces pathology to the same extent as that of $\mathrm{Tau}^{\mathrm{AT}}$ (Fig. 8e). This supports earlier findings in mice showing that tau needs to move out of the cell body in order to exert its toxic effects [48]. In the light of the current interest in the cell-to-cell spreading of Tau it would be interesting to know whether the intercellular propagation of $\mathrm{Tau}^{\mathrm{AT}}$ could contribute to the toxicity. However, this issue cannot be addressed in the current model, as mutant Tau is expressed pan-neuronally.

Taken together, the detailed description of the pathology due to the $\mathrm{Tau}^{\mathrm{AT}}$ mutation provided by the new worm models opens up multiple possibilities to identify cellular components involved in the Tau-dependent pathology and might contribute to the development of novel therapeutic interventions of tauopathies.

\section{Conclusions}

In this study, we present a new $C$. elegans model to understand the pathological consequences of A152TMAPT mutation, recently identified in patients diagnosed with frontotemporal spectrum disorders, including PSP, FTD, $\mathrm{CBD}$, and $\mathrm{AD}$. Using this model we found that, while the wild-type human tau (Tau $\left.{ }^{\mathrm{wt}}, 2 \mathrm{~N} 4 \mathrm{R}\right)$ overexpression in $C$. elegans neurons induces a progressive mild locomotor defects in a dose-dependent manner, mutant tau (Tau ${ }^{\mathrm{A} 152 \mathrm{~T}}$, 2N4R) induces a severe paralysis accompanied by acute neuronal dysfunction. Tau ${ }^{\mathrm{A} 152 \mathrm{~T}}$ worms show morphological alterations in neurons similar to what has been reported in aging neurons and exhibit a reduced life-span. $\mathrm{Tau}^{\mathrm{A} 152 \mathrm{~T}}$ overexpressing neurons show mislocalization of cellular organelles and disrupted mitochondrial trafficking. Importantly, $\mathrm{Tau}^{\mathrm{A} 152 \mathrm{~T}}$ remains in a pathological conformation but does not accumulate as insoluble aggregates. Moreover, the C-terminal domain of tau which engages with the microtubules is necessary to induce pathology despite the fact that the A152T mutation lies in the $\mathrm{N}$-terminal domain. Together these data show that the mutant $\mathrm{Tau}^{\mathrm{A} 152 \mathrm{~T}}$ overexpression in C. elegans neurons induces pathology that is not dependent on aggregation.

\section{Methods}

\section{Plasmid constructs}

To generate A152T cDNA [6], we used site-directed mutagenesis (Stratagene QuikChange reagents) to introduce the point mutation A152T into htau40WT cDNA. The respective cDNAs encoding wild-type htau40WT and mutant htau40A152T were ligated into SalI and KpnI sites of pPD49.26 vector between the snb-1 promoter and unc-54 3'-UTR (a generous gift from Dr. B.C. Kraemer, University of Washington, Seattle). Two additional proline substitutions were introduced into mutant htau40A152T in the hexapeptide motifs (I277P and I308P, intended to break beta-structure) [73]. The resulting cDNA was cloned into SalI and KpnI sites of pPD49.26 as described above, yielding Psnb-1::htau40A152T $\left(I^{277} P\right)\left(I^{308} P\right)$. Similarly, another Tau chimaera with A152T plus V337M mutation was generated as described above and cloned into SaII and KpnI sites of pPD49.26, yielding Psnb-1::htau40A152TV337M. As the A152T mutation lies outside the repeat region of Tau in the N-terminal domain, we also generated worms expressing only the $\mathrm{N}$-terminal regions of tau, residues 1243. We cloned the first 243 amino acid region of wildtype and mutant tau cDNA into the BamHI and KpnI sites of pPD49.26 to generate the transgene constructs Psnb-1::N 't-htauWT ${ }^{\text {Met1-Leu243 }}$ and Psnb-1::N't-htauA152T $T^{\text {Met1-Leu243 }}$ 
respectively. All plasmid constructs were confirmed by DNA sequencing.

\section{Generation of transgenic lines}

Complex arrays were generated by injection of Tau transgenes along with a coinjection marker (Pmyo-2::gfp or Pmyo-2::mCherry, gift of Dr. R. Baumeister) into the gonad of N2 (Bristol) at $75 \mathrm{ng} / \mu \mathrm{l}$. Coinjection markers were used at a concentration of $20 \mathrm{ng} / \mu \mathrm{l}$ and also served as controls when injected alone at the same concentrations. To generate the stable lines, the transgenes were integrated by exposing the animals to $300 \mathrm{~J} / \mathrm{m}^{2} \mathrm{UV}$ dose [74]. Multiple stable transgenic lines were isolated, and before proceeding further, the transgenic lines were backcrossed to N2 wild-type at least five times to eliminate any background mutations.

C. elegans maintenance and crosses were performed according to the standard methods [75]. Worms were cultured at $20{ }^{\circ} \mathrm{C}$ unless otherwise specified. N2 Bristol was used as the wild-type $C$. elegans. The following transgenic strains were used: PIR1: pirEx1[Psnb-1::htau40WT;Pmyo2::gfp], PIR2: pirEx2[Psnb-1::htau40A152T;Pmyo-2::gfp], PIR3: pirIs3[Psnb-1::htau40WT-low;Pmyo-2::gfp], PIR4: pirIs4[Psnb-1::htau40WT-high;Pmyo-2::gfp], PIR5: pirIs5[Psnb1::htau40A152T-low;Pmyo-2::gfp], PIR6: pirIs6[Psnb-1::hta u40A152T-high;Pmyo-2::gfp], PIR7: pirEx7[Psnb-1::htau40A 152T $\left(I^{277} P\right)\left(I^{308} P\right)$;Pmyo-2::mCherry], PIR8: pirEx8[Psnb1::N't-tauWT Met1-Leu243;Pmyo-2::mCherry], PIR9: pirEx9[Psnb-1::N't-tauA152T Met 1-Leu243;Pmyo-2::mCherry], CZ1197: juIs73[Punc-25:::gfp]III (gift of Dr. E. Lundquist), PIR10: pirIs3;juIs73, PIR11: pirIs4;juIs73, PIR12: pirIs5;juIs73, PIR13: pirIs6;juIs73, zdIs5:[Pmec-4::GFP + lin-15(+)], PIR14: pirIs3;zdIs5, PIR15: pirIs4;zdIs5, PIR16: pirIs5;zdIs5, vdEx262:[Pmec-4::mCherry::rab-3;Punc122::gfp] (gift of Dr. M. Hilliard), PIR17: pirIs3;vdEx262, PIR18: pirIs4; vdEx262, PIR19: pirIs5;vdEx262, wyEx2709: [Pitr-1::TOM-20154aa::yfp;Podr-1::gfp] (gift of Dr. K. Shen), PIR20: pirIs3;wyEx2709, PIR21: pirIs4;wyEx2709, PIR22: pirIs5;wyEx2709, jsIs609:Is:[Pmec-4::MLS::gfp] (gift of Dr. Nonet M), PIR23: pirIs3; jsIs609, PIR24: pirIs4; jsIs609, PIR25: pirIs5;jsIs609, kyIs445:Is:[Pdes-2::mCherry:::RAB-3;des-2::SAD-1::GFP;odr1::DsRED] (gift of Dr. C.I. Bargmann), PIR26: pirIs3;kyIs445, PIR27: pirIs4;kyIs445, PIR28: pirIs5;kyIs445, PIR29: pirEx29 [Psnb-1::htau40A152TV337M;Pmyo-2::mCherry], CK10: bkIs10[Paex-3::htauV337M(1N4R);Pmyo-2::gfp], CB211: lev1(e211)IV and NM791: rab-3(js49)II.

\section{Behavioral assays}

For qualitative locomotor behavior, synchronized day-1 old adults were placed onto the center of NGM plates freshly spotted with E. coli OP-50 and photographed after $10 \mathrm{~min}$. For thrash assays, synchronized animals from each transgenic line were transferred into $20 \mu \mathrm{l}$ of M9 buffer (22 mM KH $\mathrm{HO}_{4}, 42 \mathrm{mM} \mathrm{Na} \mathrm{HPO}_{4}, 86 \mathrm{mM}$
$\mathrm{NaCl}$ and $1 \mathrm{mM} \mathrm{MgSO}_{4}$ ) on a glass slide. After allowing the animals to settle for $1 \mathrm{~min}$, the frequency of body bending was counted for $30 \mathrm{~s}$ [76].

\section{Life span assay}

A synchronous population of worms was obtained by extracting eggs from gravid adults and incubated in M9 buffer overnight. Synchronised L1 larvae were collected and allowed to grow until late L4 stage on regular NGM plates seeded with E. coli OP50, and then transferred onto OP50 seeded NGM plates containing $0.05 \mathrm{mg} / \mathrm{ml}$ FUDR (5-fluoro-2-deoxyuridine) for the first two days to inhibit growth of progeny. Thereafter, the worms were grown again on regular OP50 seeded NGM plates and scored every two days by tugging gently on the tail with a platinum wire covered with bacteria. Worms that failed to respond to touch were scored as dead. GraphPad Prism software was used to perform the statistical analysis.

\section{Immunohistochemistry}

Worms were permeabilized by freeze cracking after fixation in $2 \%$ paraformaldehyde solution as described previously [58]. Polyclonal rabbit pan-tau antibody (K9JA; Dako A-0024) at a dilution of 1:5000 was used to detect Tau. To monitor the conformational changes, we used the pathological conformation-specific monoclonal mouse antibody MC-1 [77]; (gift from Dr. P. Davies; 1:20). For immunostaining of worms expressing N-terminal tau fragments, rabbit polyclonal antibody SA4473 (Eurogentec; 1:300) was used. Respective secondary antibodies were used at 1:350 dilutions. Stained animals were imaged at 63x using a Zeiss inverted epifluorescence microscope.

\section{Imaging}

GABAergic motor neurons were imaged by mounting immobilized young adult animals in $50 \mathrm{mM}$ sodium azide (Sigma) on glass slides with $2 \%$ agarose pads, and imaged using a 20x or 40x objective on LSM700 (Zeiss). To study the steady state distribution of synaptic vesicles, worms were anesthetized in $50 \mathrm{mM}$ sodium azide, mounted on $2 \%$ agarose pads, and neurons were imaged at $63 \mathrm{x}$ with Zeiss inverted epifluorescence microscope. For steady state imaging of mitochondrial marker Pitr1:TOM-20 $0^{1-54 a a}:: y f p$, we used Nikon microscope equipped with Andor Spinning Disc Setup and EM-CCD camera (Andor iXon 3). For live imaging of GFP tagged mitochondria, worms of appropriate age (day- 1 and day3 old adult) were anesthetized in $2-3 \mathrm{mM}$ of levamisole (Biomol) [58] and mounted on $2 \%$ agarose pads. Time lapse (3fps) images of mitochondria tagged with GFP in mechanosensory neurons were acquired at $63 \mathrm{x}$ with a Zeiss epifluorescence microscope equipped with a CCD (Photometrics) camera. Images were acquired for 
constant time $(\mathrm{t}=2.5 \mathrm{~min})$ in two different regions of a neuron, in the proximal part (axonal part adjacent to cell body, $\sim 60-80 \mu \mathrm{m}$ ) and middle part $\sim 120 \mu \mathrm{m}$ away from cell body (refer to schematic in Fig. 4a). The number of moving mitochondrial events was counted manually for each genotype from the acquired movies. Representative kymographs were generated using an ImageJ (NIH) plug in.

\section{Aldicarb and levamisole assays}

To perform the aldicarb (acetylcholine esterase inhibitor) and levamisole (acetylcholine receptor agonist) sensitivity assays, day-1 old adult worms were transferred to plates containing $1 \mathrm{mM}$ aldicarb and $0.2 \mathrm{mM}$ levamisole, respectively and the time course of paralysis was assayed as described previously [36, 78]. 20 animals per strain were scored for paralysis and each experiment was repeated three times.

\section{Pharmacological treatment}

Chemical treatment was applied in liquid cultures as described earlier [17]. Aggregation inhibitor compounds bb14 (Rhodanine) or BSc3094 (PTH) were dissolved in DMSO and added to the liquid culture at 50 and $100 \mu \mathrm{M}$ concentrations.

\section{Protein extraction}

To extract total Tau protein, synchronized worms were washed off NGM plates with water and the bacteria were removed in the subsequent washing steps. The resulting worm pellets $(\sim 100 \mathrm{mg})$ were resuspended in $1 \mathrm{X}$ protein sample buffer containing $355 \mathrm{mM}$ 2-mercaptoethanol and boiled at $96{ }^{\circ} \mathrm{C}$ for $10 \mathrm{~min}$. with continuous shaking at $14,000 \mathrm{rpm}$. To determine the accumulation of insoluble tau aggregates, a slight modification of the previous extraction protocol [17] was performed using buffers of increasing stringency. Briefly, after removing the dead animals and bacteria by flotation on a $30 \%$ sucrose solution, worm pellets $(\sim 100 \mathrm{mg})$ were resuspended in twice the amount (w/v) of high-salt RAB buffer [100 mM 2(N-morpholino) ethanesulfonic acid (MES), $1 \mathrm{mM}$ EGTA, $0.5 \mathrm{mM} \mathrm{MgSO}_{4}, 20 \mathrm{mM} \mathrm{NaF}$. Worm pellets were lysed completely by sonication $(6 \times 10 \mathrm{~s}, 10 \mathrm{~s}$ break) on ice, and homogenates centrifuged at 40,000 g for $40 \mathrm{~min}$. The supernatant is the soluble RAB fraction. $\mathrm{RAB}$ pellet was sonicated for $10 \mathrm{~s}$ in $1 \mathrm{M}$ sucrose containing $\mathrm{RAB}$ buffer and centrifuged for $20 \mathrm{~min}$ at $40,000 \mathrm{~g}$, and the supernatant was discarded. The pellet was extracted with RIPA buffer (150 mM NaCl, $1 \%$ Nonidet P-40, $0.5 \%$ deoxycholate, $0.1 \%$ SDS, $50 \mathrm{mM}$ Tris, $\mathrm{pH}$ 8.0) and centrifuged at 40,000 g for $20 \mathrm{~min}$. The supernatant constitutes the detergent soluble RIPA fraction. The resulting pellet, after a brief washing with RIPA buffer, was extracted with urea containing buffer
(UREA) [30 mM Tris, $7 \mathrm{M}$ urea, $2 \mathrm{M}$ thiourea, $4 \%$ CHAPS (3-[(3-cholamidopropyl)dimethylammonio]-1propanesulfonate), $\mathrm{pH}$ 8.5] and centrifuged at 13,000 g for $15 \mathrm{~min}$. The supernatant is the detergent insoluble fraction.

For native PAGE, frozen worm pellets $(\sim 100 \mathrm{mg})$ were resuspended in buffer $\mathrm{C}$ (20 mM Hepes, $\mathrm{pH} 7.9,25 \%$ glycerol, $0.42 \mathrm{M} \mathrm{NaCl}, 1.5 \mathrm{mM} \mathrm{MgCl}_{2}, 0.2 \mathrm{mM}$ EDTA, $0.5 \mathrm{mM}$ DTT $)$ and lysed by sonication $(2 \times 10 \mathrm{~s}, 10 \mathrm{~s}$ break) on ice. After a brief centrifugation at 40,000 $\mathrm{g}$ for $5 \mathrm{~min}$, the supernatants were analyzed on 4-12\% native PAGE. The entire extraction procedures were carried out on ice and centrifugation steps were at $4{ }^{\circ} \mathrm{C}$. All buffers contained Complete Protease Inhibitor Mixture 3× (Sigma-Aldrich P8340, Hamburg, Germany), $1 \mu \mathrm{M}$ okadaic acid (phosphatase inhibitor) and $0.5 \mathrm{mM}$ PMSF (protease inhibitor).

\section{Supplementary methods Immunoblotting}

Worm fractions (total or sequentially extracted - salt soluble, detergent soluble or detergent insoluble) were migrated in $10 \%$ polyacrylamide gels, transferred to nitrocellulose membranes (Immobilon) and immunoblotted. The following antibodies were used: DM1 $\alpha$ tubulin (1:500; Sigma), K9JA (1:20,000; no. A0024; Dako), AT8 (1:500; Thermo Scientific), PHF-1 (1:500; a gift from Dr. P. Davies, Albert Einstein College, Bronx, USA), 12E8 (1:500; Dr. P. Seubert, Elan Pharmaceuticals, South San Francisco, USA), AT180 (1:500; Thermo Scientific), AT100 (1:500; Thermo Scientific). For worms expressing the $\mathrm{N}$-terminal region of tau, antibody DA9 (raised against aa100-130, a gift from Dr. P. Davies) was used at a dilution of 1:250. Peroxide-conjugated secondary antibodies and ECL solution (Thermo Scientific) were used to visualize the blots. AIDA software (Raytest, Germany) was used to perform densitometry.

\section{Additional files}

Additional file 1: Movie S1. Thrash assay performed with day-1 old Tau $^{\text {wt }}$-lo in M9 buffer. (MOV 3770 kb)

Additional file 2: Movie S2. Thrash assay performed with day-1 old Tau ${ }^{\text {wt }}$-hi in M9 buffer. (MOV 6133 kb)

Additional file 3: Movie S3. Thrash assay performed with day-1 old Tau ${ }^{\text {AT }}$-lo in M9 buffer. (MOV 3920 kb)

Additional file 4: Movie S4. Thrash assay performed with day-1 old Tau ${ }^{\text {AT }}$-hi in M9 buffer. (MOV 3326 kb)

Additional file 5: Movie S5. Representative time lapse imaging showing the movement of MLS::GFP labelled mitochondria in touch neuron of a non-tg. The cell bodies in all the movies (not visible) are to the left. White arrow marks anterogradely and pink retrogradely moving mitochondria. Movies are displayed at 10x speed, scale bar: $5 \mu \mathrm{m}$. (MOV 432 kb) 
Additional file 6: Movie S6. Representative time lapse imaging showing the movement of MLS::GFP labelled mitochondria in touch neuron of a Tau'st-lo. (MOV 200 kb)

Additional file 7: Movie S7. Representative time lapse imaging showing the movement of MLS::GFP labelled mitochondria in touch neuron of a Tau'twi.hi. (MOV $311 \mathrm{~kb}$ )

Additional file 8: Movie S8. Representative time lapse imaging showing the movement of MLS::GFP labelled mitochondria in touch neuron of a Tau ${ }^{\mathrm{AT}}-\mathrm{lo}$. (MOV $255 \mathrm{~kb}$ )

Additional file 9: Pan-neuronal expression of human tau in C. elegans by synaptobrevin promoter. Immunochemistry of Tau ${ }^{\text {wt }}$-hi and its mutant counterpart Tau ${ }^{\text {AT }}$-hi with pan-tau antibody K9JA showing Tau staining in the nervous system. Panels on the left depict nerve ring ganglion, panels on the right side show the ventral cord region. K9JA pan-tau antibody does not stain wild-type non-tg worms (data not shown). (PDF 110 kb)

Additional file 10: Mutant Tau ${ }^{\mathrm{AT}}$ transgene present as

extrachromosomal arrays induces severe locomotion defects in C. elegans. (A) Worms carrying human tau-transgene arrays with A152T mutation Ex[Tau $\left.{ }^{\mathrm{AT}}\right]$ show a severe motor impairment compared to the worms carrying wild-type human tau-transgene arrays Ex[Tau $\left.{ }^{\text {wt }}\right]$. Worms were allowed to crawl for 10 min after placing them onto freshly spotted NGM plates before photographing them. Ex[Tau $\left.{ }^{\mathrm{AT}}\right]$ worms are highly uncoordinated and show a severe paralytic phenotype obvious by the absence of tracks in the E. coli lawn and coiling behaviour. Arrows point to the displacement from the origin. (B) 30 day-1 adult animals from non-tg, Ex[Tau $\left.{ }^{\mathrm{wt}}\right]$ and Ex[Tau $\left.{ }^{\mathrm{AT}}\right]$ lines were lysed and the lysate was subjected to $10 \%$ PAGE and subsequent western blot analysis using K9JA-pan-tau antibody. Both Ex[Tau $\left.{ }^{\text {wt }}\right]$ and Ex[Tau $\left.{ }^{\mathrm{AT}}\right]$ carrying tau transgenes as extrachromosomal arrays show comparable expression of human tau. (C) Mean thrashing rate of day-1 old animals from strains carrying Tau ${ }^{\text {wt }}$ and Tau ${ }^{\mathrm{AT}}$ transgenes as extrachromosomal arrays. Non-tg wild-type strain serves as control. Error bars denote SEM, $n \geq 30$. ${ }^{*} P<0.05$, ${ }^{* *} P<0.01$, ${ }^{* *} P<0.001$. One-way ANOVA with Tukey's test was applied for multiple comparisons. (D) Punc-25::gfp reporter staining GABAergic motor neurons with GFP in non-tg and Tau-transgenic worms. Non-tg control worms show intact ventral and dorsal nerve cords. Transgenic worms

carrying Tau-transgenes as extrachromosomal arrays show abnormal GABAergic motor neurons. Worms expressing mutant htau40A152T (Ex[Tau $\left.{ }^{\mathrm{AT}}\right]$ ) show a severe loss of GABAergic neuronal system compared to the worms expressing wild-type tau (Ex[Tau ${ }^{\text {wt }}$ ). Note the gaps (arrow heads) and absence of dorsal cord stretches (bracketed region) in Ex[Tau $\left.{ }^{\mathrm{AT}}\right]$ worms. (PDF $683 \mathrm{~kb}$ )

Additional file 11: Expression of mutant Tau ${ }^{\text {AT }}$ produces a substantial damage in GABAergic motor neurons. Representative whole worm maximum intensity projections (MIP) of the enlarged insets presented in main Fig. 2. Punc-25::gfp reporter that labels GABAergic inhibitory neurons with GFP, was crossed with respective tau-transgenic worms to visualize these neurons. GABAergic neurons show normal connectivity in non-tg worms, both dorsal and ventral nerve cords are intact (A). Expression of $\mathrm{Tau}^{\mathrm{wt}}$ produced dose dependent abnormalities in GABAergic neurons, with Tau ${ }^{\text {wt }}$-hi neurons (C) accumulating more damage than Tau ${ }^{\text {wt }}-$ lo (B). However, mutant Tau ${ }^{\mathrm{AT}}$ expression led to severe abnormalities in the form of gaps (arrowheads) in the dorsal and ventral nerve cords, in both Tau $^{\text {AT }}$-lo (D) and Tau ${ }^{\text {AT }}$-hi (E). Also see Table 1 for detailed quantitative analysis. (PDF $276 \mathrm{~kb}$ )

Additional file 12: Aberrant localization of presynaptic components in mechanosensory neurons worsens with age in mutant Tau ${ }^{\text {AT }}$ worms. (A) Schematic representation of presynaptic cargo distribution in a normal healthy mechanosensory neuron and in an aging neuron. (B) Presynaptic cargo visualized in day-3 old worms after crossing them into vdEx262:[Pmec-4:.mCherry::rab-3] transgene, that expresses mCherry fused to synaptic vesicle associated RAB-3 in mechanosensory neurons. Mislocalization worsens in Tau ${ }^{\text {AT }}$-lo, whereas Tau ${ }^{\text {wt }}$-lo and Tau ${ }^{\text {wt }}$-hi start accumulating mCherry::RAB-3 puncta in the distal axon (yellow arrowheads) and posterior neurite (white arrow). At day 3 , the mid neuron of A152T worms becomes almost devoid of mCherry::RAB3 puncta. (PDF $186 \mathrm{~kb})$

Additional file 13: Mutant Tau ${ }^{\mathrm{AT}}$ does not aggregate in C. elegans neurons. (A) Sequential extraction reveals no insoluble tau in day-1 old adults. Tau was sequentially extracted from day-1 old transgenic worms, wild-type N2 (Bristol) worms served as control. Worms were lysed by sonication in high-salt buffer (RAB), centrifuged and supernatant collected as salt soluble Tau fraction. Reextraction of salt insoluble pellet with detergent buffer (RIPA) yielded detergent soluble Tau fraction. RIPA insoluble pellet was finally dissolved in urea buffer (UREA) to extract any insoluble fraction. Equal amount of protein was loaded and blotted using K9JA pan-tau antibody. (B) Bar diagram of Tau construct Tau ${ }^{A T+V M}$ with A152T mutation plus additional methionine substitution in the repeat domain (V337M) (htau40A152TV337M). (C) 30 day-1 adult animals from non-tg, Ex[Tau $\left.{ }^{\mathrm{AT}}\right]$ and the double mutant variant Ex[Tau $\left.{ }^{\mathrm{AT}+\mathrm{VM}}\right]$ after lysis in 1 $x$ sample buffer, then subjected to $10 \%$ PAGE and subsequent western blot analysis using K9JA-pan-tau antibody. Ex[Tau $\left.{ }^{\mathrm{AT}}\right]$ and Ex[Tau $\left.{ }^{\mathrm{AT}+\mathrm{VM}}\right]$ carrying the respective Tau transgenes as extrachromosomal arrays (Ex) show comparable levels of Tau expression. Tubulin serves as loading control. (D) Mean thrashing assay of day-1 old adult animals carrying Tau ${ }^{\text {AT }}$ or the double mutant variant (Tau ${ }^{\mathrm{AT}+\mathrm{VM}}$ ) transgenes as extrachromosomal arrays. Ex[Tau $\left.{ }^{\mathrm{AT}+\mathrm{VM}]}\right]$ shows less thrashes than the non-tg ( 5\% of non-tg). But there is no difference between the single mutant variant Ex[Tau $\left.{ }^{A T}\right]$ and the double mutant variant Ex[Tau $\left.{ }^{\mathrm{AT}+\mathrm{VM}}\right]$. Non-tg strain serves as control. Error bars denote SEM, $n \geq 30$, ns., not significant. One-way ANOVA with Tukey's test applied for multiple comparisons. (PDF $246 \mathrm{~kb}$ )

Additional file 14: Mutant Tau ${ }^{\text {AT }}$ does not differ in phosphorylation status from wild-type Tau (Tau $\left.{ }^{w t}\right)$. Total Tau extracted from tau-tg worms was examined for the phosphorylation status at the sites known to be highly phosphorylated in the AD brain. Western blot analysis with phosphorylation specific antibodies show that tau in all the strains is highly phosphorylated at multiple sites examined (AT8 for pS199, pS202, and PT205; PHF-1 for pS396 and pS404; 12 E8 for pS262 and pS356; AT180 for pT231 and pS235, AT100 for pT212 and pS214). In addition to the monomeric tau band ( $\approx 64 \mathrm{KDa}$, single arrow head), the phosphorylation specific AT8 antibody also detects a band slightly higher than $64 \mathrm{KDa}$ band (double arrow head) and another band of $\sim 120-K D a$ (triple arrow head) specific only to tau-transgenic worms. BV P20 = highly phosphorylated Tau of Baculovirus infected SF9 cells [79]. (PDF $355 \mathrm{~kb}$ )

Additional file 15: Mutant tau with anti-aggregant proline substitutions

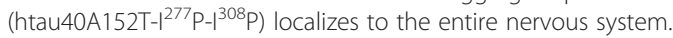
Immunohistochemistry of whole worms with pan-tau K9JA antibody shows Tau ${ }^{\text {AT+PP }}$ localization in cell body and the neuronal processes. Panels on the left side depict nerve ring ganglion and those on the right side show ventral cord region. (PDF $174 \mathrm{~kb}$ )

\section{Abbreviations}

1N4R-Tau: 412 residues, 1 insert +4 repeats; 2N4R-Tau: largest isoform of human tau in brain (441 residues, 2 inserts +4 repeats); ANOVA: analysis of variance; MAPT: microtubule associated protein tau; MT: microtubules; nontg: non-transgenic; PHF: paired helical filaments; Tau ${ }^{\mathrm{AT}}$ : human Tau (2N4R) with mutation A152T; Tau ${ }^{\text {AT+PP }}$ : human Tau (2N4R) with proline substitutions in the hexapeptide motifs; Tau ${ }^{\text {AT }+V M}$ : human Tau (2N4R) with mutation

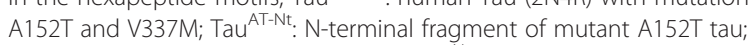
Tau' ${ }^{\text {wt. }}$ human wild type Tau (2N4R); Tau ${ }^{\text {wt-Nt: }}$ N-terminal fragment of wildtype tau; Unc: uncoordinated locomotion.

\section{Competing interests}

The authors declare that they have no competing interests.

\section{Author's contributions}

GJP, EM and EMM conceived and designed the experiments. GJP and BC performed research. GJP, BC, EM and EMM analyzed data; GJP, EM and EMM wrote the paper with inputs from BC. All authors have read and approved the final version of the manuscript.

\section{Acknowledgements}

We are highly grateful to Daniele Bano (DZNE, Bonn) for allowing us to use his lab equipment and for critical reading of the manuscript. His advice and continued support throughout the course of this work has been invaluable. We thank the following colleagues for providing strains and reagents crucial for this study: Adam Antebi (Max-Planck Institute for Biology of Ageing, Cologne, Germany), Brian Kraemer (University of Washington, Seattle, USA), Erik Lundquist (University of Kansas, USA), Kang Shen (Stanford University, 
USA), Massimo Hilliard (University of Queensland, Australia), Michael Nonet (Washington University in St. Louis), Peter Davies (Feinstein Institute for Medical Research, North Shore LIJ, USA), Ralf Baumeister (Institute of Biology III, Freiburg, Germany). We are also grateful to Jacek Biernat (DZNE, Bonn), DZNE Light Microscope Facility (Hans Fried, Irene König) and Laboratory Management (Max Koistinen, Paula Urrutia, Stefanie Nickolaus). Some strains were provided by the CGC, funded by $\mathrm{NIH}$ Office of Research Infrastructure Programs (P40 OD010440).

This work was supported by the German Center for Neurodegenerative Diseases, Max-Planck-Society, Tau Consortium, Wellcome Trust/MRC Neurodegenerative Diseases Initiative, and Katharina-Hardt-Foundation.

\section{Author details}

${ }^{1}$ German Center for Neurodegenerative Diseases (DZNE), Ludwig-Erhard-Allee 2, 53175 Bonn, Germany. ${ }^{2}$ Caesar Research Center, Ludwig-Erhard-Allee 2, 53175 Bonn, Germany. ${ }^{3}$ Max-Planck-Institute for Metabolism Research (Cologne), Hamburg Outstation, c/o DESY, Notkestrasse 85, 22607 Hamburg, Germany.

\section{Received: 18 December 2015 Accepted: 8 April 2016} Published online: 27 April 2016

\section{References}

1. Goedert M, Crowther RA, Spillantini MG. Tau mutations cause frontotemporal dementias. Neuron. 1998;21(5):955-8.

2. Grundke-lqbal I, labal K, Tung YC, Quinlan M, Wisniewski HM, Binder LI. Abnormal phosphorylation of the microtubule-associated protein tau (tau) in Alzheimer cytoskeletal pathology. Proc Natl Acad Sci U S A. 1986;83(13):4913-7.

3. Mandelkow EM, Mandelkow E. Biochemistry and cell biology of tau protein in neurofibrillary degeneration. Cold Spring Harb Perspect Med. 2012;2(7): a006247. doi:10.1101/cshperspect.a006247.

4. McKhann GM, Albert MS, Grossman M, Miller B, Dickson D, Trojanowski JQ. Clinical and pathological diagnosis of frontotemporal dementia: report of the Work Group on Frontotemporal Dementia and Pick's Disease. Arch Neurol. 2001;58(11):1803-9.

5. von Bergen M, Barghorn S, Biernat J, Mandelkow EM, Mandelkow E. Tau aggregation is driven by a transition from random coil to beta sheet structure. Biochim Biophys Acta. 2005;1739(2-3):158-66.

6. Coppola G, Chinnathambi S, Lee JJ, Dombroski BA, Baker MC, Soto-Ortolaza $\mathrm{Al}$, et al. Evidence for a role of the rare p.A152T variant in MAPT in increasing the risk for FTD-spectrum and Alzheimer's diseases. Hum Mol Genet. 2012;21(15):3500-12. doi:10.1093/hmg/dds161.

7. Kara E, Ling H, Pittman AM, Shaw K, de Silva R, Simone R, et al. The MAPT p.A152T variant is a risk factor associated with tauopathies with atypical clinical and neuropathological features. Neurobiol Aging. 2012;33(9):2231 e7- e14. doi:10.1016/j.neurobiolaging.2012.04.006.

8. Kovacs GG, Wohrer A, Strobel T, Botond G, Attems J, Budka H. Unclassifiable tauopathy associated with an A152T variation in MAPT exon 7. Clin Neuropathol. 2011;30(1):3-10.

9. Morris M, Maeda S, Vossel K, Mucke L. The many faces of tau. Neuron. 2011; 70(3):410-26. doi:10.1016/j.neuron.2011.04.009.

10. Hanger DP, Lau DH, Phillips EC, Bondulich MK, Guo T, Woodward BW, et al. Intracellular and extracellular roles for tau in neurodegenerative disease. J Alzheimers Dis. 2014;40 Suppl 1:S37-45. doi:10.3233/JAD-132054.

11. Alexander AG, Marfil V, Li C. Use of Caenorhabditis elegans as a model to study Alzheimer's disease and other neurodegenerative diseases. Front Genet. 2014:5:279. doi:10.3389/fgene.2014.00279.

12. Therrien M, Parker JA. Worming forward: amyotrophic lateral sclerosis toxicity mechanisms and genetic interactions in Caenorhabditis elegans. Front Genet. 2014;5:85. doi:10.3389/fgene.2014.00085.

13. Schmidt E, Seifert M, Baumeister R. Caenorhabditis elegans as a model system for Parkinson's disease. Neurodegener Dis. 2007;4(2-3):199-217. doi: 10.1159/000101845.

14. Shaye DD, Greenwald I. OrthoList: a compendium of C. elegans genes with human orthologs. PLoS One. 2011;6(5):e20085. doi:10.1371/journal.pone. 0020085.

15. Kraemer BC, Schellenberg GD. SUT-1 enables tau-induced neurotoxicity in C. elegans. Hum Mol Genet. 2007;16(16):1959-71. doi:10.1093/hmg/ddm143.

16. Guthrie CR, Schellenberg GD, Kraemer BC. SUT-2 potentiates tau-induced neurotoxicity in Caenorhabditis elegans. Hum Mol Genet. 2009;18(10): 1825-38. doi:10.1093/hmg/ddp099.
17. Fatouros C, Pir GJ, Biernat J, Koushika SP, Mandelkow E, Mandelkow EM, et al. Inhibition of tau aggregation in a novel Caenorhabditis elegans model of tauopathy mitigates proteotoxicity. Hum Mol Genet. 2012;21(16): 3587-603. doi:10.1093/hmg/dds190.

18. Wischik CM, Staff RT, Wischik DJ, Bentham P, Murray AD, Storey JM, et al. Tau aggregation inhibitor therapy: an exploratory phase 2 study in mild or moderate Alzheimer's disease. J Alzheimers Dis. 2015;44(2):705-20. doi:10. 3233/JAD-142874.

19. Schuske K, Beg AA, Jorgensen EM. The GABA nervous system in C. elegans. Trends Neurosci. 2004;27(7):407-14. doi:10.1016/j.tins.2004.05.005.

20. Lundquist EA, Reddien PW, Hartwieg E, Horvitz HR, Bargmann Cl. Three C. elegans Rac proteins and several alternative Rac regulators control axon guidance, cell migration and apoptotic cell phagocytosis. Development. 2001;128(22):4475-88.

21. Knobloch M, Mansuy IM. Dendritic spine loss and synaptic alterations in Alzheimer's disease. Mol Neurobiol. 2008;37(1):73-82. doi:10.1007/s12035008-8018-z.

22. Yankner BA, Lu T, Loerch P. The aging brain. Annu Rev Pathol. 2008;3:41-66. doi:10.1146/annurev.pathmechdis.2.010506.092044.

23. Pan $\mathrm{CL}$, Peng $\mathrm{CY}$, Chen $\mathrm{CH}$, Mclntire S. Genetic analysis of age-dependent defects of the Caenorhabditis elegans touch receptor neurons. Proc Natl Acad Sci U S A. 2011;108(22):9274-9. doi:10.1073/pnas.1011711108.

24. Toth ML, Melentijevic I, Shah L, Bhatia A, Lu K, Talwar A, et al. Neurite sprouting and synapse deterioration in the aging Caenorhabditis elegans nervous system. J Neurosci. 2012;32(26):8778-90. doi:10.1523/JNEUROSCI. 1494-11.2012.

25. Apfeld J, Kenyon C. Regulation of lifespan by sensory perception in Caenorhabditis elegans. Nature. 1999;402(6763):804-9. doi:10.1038/45544.

26. Alcedo J, Kenyon C. Regulation of C. elegans longevity by specific gustatory and olfactory neurons. Neuron. 2004;41(1):45-55.

27. Wolkow CA, Kimura KD, Lee MS, Ruvkun G. Regulation of C. elegans lifespan by insulinlike signaling in the nervous system. Science. 2000;290(5489): 147-50.

28. Iser WB, Gami MS, Wolkow CA. Insulin signaling in Caenorhabditis elegans regulates both endocrine-like and cell-autonomous outputs. Dev Biol. 2007; 303(2):434-47. doi:10.1016/j.ydbio.2006.04.467.

29. Antebi A. Ageing: when less is more. Nature. 2007;447(7144):536-7. doi:10. 1038/447536a.

30. Barkus RV, Klyachko O, Horiuchi D, Dickson BJ, Saxton WM. Identification of an axonal kinesin-3 motor for fast anterograde vesicle transport that facilitates retrograde transport of neuropeptides. Mol Biol Cell. 2008;19(1): 274-83. doi:10.1091/mbc.E07-03-0261.

31. Nonet ML, Staunton JE, Kilgard MP, Fergestad T, Hartwieg E, Horvitz HR, et al. Caenorhabditis elegans rab-3 mutant synapses exhibit impaired function and are partially depleted of vesicles. J Neurosci. 1997;17(21):8061-73.

32. Neumann B, Hilliard MA. Loss of MEC-17 leads to microtubule instability and axonal degeneration. Cell Rep. 2014;6(1):93-103. doi:10.1016/j.celrep. 2013.12.004

33. Albeg A, Smith CJ, Chatzigeorgiou M, Feitelson DG, Hall DH, Schafer WR et al. C. elegans multi-dendritic sensory neurons: morphology and function. Mol Cell Neurosci. 2011;46(1):308-17. doi:10.1016/j.mcn.2010.10.001.

34. Gioran A, Nicotera P, Bano D. Impaired mitochondrial respiration promotes dendritic branching via the AMPK signaling pathway. Cell Death Dis. 2014;5: e1175. doi:10.1038/cddis.2014.144.

35. Maniar TA, Kaplan M, Wang GJ, Shen K, Wei L, Shaw JE, et al. UNC-33 (CRMP) and ankyrin organize microtubules and localize kinesin to polarize axon-dendrite sorting. Nat Neurosci. 2012;15(1):48-56. doi:10.1038/nn.2970.

36. Mahoney TR, Luo S, Nonet ML. Analysis of synaptic transmission in Caenorhabditis elegans using an aldicarb-sensitivity assay. Nat Protoc. 2006; 1(4):1772-7. doi:10.1038/nprot.2006.281.

37. Klassen MP, Wu YE, Maeder CI, Nakae I, Cueva JG, Lehrman EK, et al. An Arf-like small G protein, ARL-8, promotes the axonal transport of presynaptic cargoes by suppressing vesicle aggregation. Neuron. 2010;66(5):710-23. doi: 10.1016/j.neuron.2010.04.033

38. Spires-Jones TL, de Calignon A, Meyer-Luehmann M, Bacskai BJ, Hyman BT. Monitoring protein aggregation and toxicity in Alzheimer's disease mouse models using in vivo imaging. Methods. 2011;53(3):201-7. doi:10.1016/j. ymeth.2010.12.009.

39. Caughey B, Lansbury PT. Protofibrils, pores, fibrils, and neurodegeneration: separating the responsible protein aggregates from the innocent 
bystanders. Annu Rev Neurosci. 2003;26:267-98. doi:10.1146/annurev.neuro. 26.010302.081142.

40. Uversky VN. Targeting intrinsically disordered proteins in neurodegenerative and protein dysfunction diseases: another illustration of the $\mathrm{D}(2)$ concept. Expert Rev Proteomics. 2010;7(4):543-64. doi:10.1586/epr.10.36.

41. Kraemer BC, Zhang B, Leverenz JB, Thomas JH, Trojanowski JQ, Schellenberg GD. Neurodegeneration and defective neurotransmission in a Caenorhabditis elegans model of tauopathy. Proc Natl Acad Sci U S A. 2003; 100(17):9980-5. doi:10.1073/pnas.1533448100.

42. von Bergen M, Friedhoff P, Biernat J, Heberle J, Mandelkow EM, Mandelkow E. Assembly of tau protein into Alzheimer paired helical filaments depends on a local sequence motif ((306)VQIVYK(311)) forming beta structure. Proc Natl Acad Sci U S A. 2000;97(10):5129-34.

43. Eckermann K, Mocanu MM, Khlistunova I, Biernat J, Nissen A, Hofmann A, et al. The beta-propensity of Tau determines aggregation and synaptic loss in inducible mouse models of tauopathy. J Biol Chem. 2007;282(43):3175565. doi:10.1074/jbc.M705282200.

44. Jicha GA, Berenfeld B, Davies P. Sequence requirements for formation of conformational variants of tau similar to those found in Alzheimer's disease. J Neurosci Res. 1999;55(6):713-23. doi:10.1002/(SICl)1097-4547(19990315)55: 6<713::AID-JNR6>3.0.CO;2-G.

45. Butner KA, Kirschner MW. Tau protein binds to microtubules through a flexible array of distributed weak sites. J Cell Biol. 1991;115(3):717-30.

46. Goedert M, Wischik CM, Crowther RA, Walker JE, Klug A. Cloning and sequencing of the CDNA encoding a core protein of the paired helical filament of Alzheimer disease: identification as the microtubule-associated protein tau. Proc Natl Acad Sci U S A. 1988;85(11):4051-5.

47. Magnani E, Fan J, Gasparini L, Golding M, Williams M, Schiavo G, et al. Interaction of tau protein with the dynactin complex. EMBO J. 2007;26(21): 4546-54. doi:10.1038/sj.emboj.7601878.

48. Ittner LM, Ke YD, Delerue F, Bi M, Gladbach A, van Eersel J, et al. Dendritic function of tau mediates amyloid-beta toxicity in Alzheimer's disease mouse models. Cell. 2010;142(3):387-97. doi:10.1016/j.cell.2010.06.036.

49. Williamson R, Scales T, Clark BR, Gibb G, Reynolds CH, Kellie S, et al. Rapid tyrosine phosphorylation of neuronal proteins including tau and focal adhesion kinase in response to amyloid-beta peptide exposure: involvement of Src family protein kinases. J Neurosci. 2002;22(1):10-20.

50. Jeganathan S, Hascher A, Chinnathambi S, Biernat J, Mandelkow EM, Mandelkow E. Proline-directed pseudo-phosphorylation at AT8 and PHF1 epitopes induces a compaction of the paperclip folding of Tau and generates a pathological (MC-1) conformation. J Biol Chem. 2008;283(46): 32066-76. doi:10.1074/jbc.M805300200.

51. Lee SE, Tartaglia MC, Yener G, Genc S, Seeley WW, Sanchez-Juan P, et al. Neurodegenerative disease phenotypes in carriers of MAPT p.A152T, a risk factor for frontotemporal dementia spectrum disorders and Alzheimer disease. Alzheimer Dis Assoc Disord. 2013;27(4):302-9. doi:10.1097/WAD. 0b013e31828cc357.

52. Gauthier-Kemper A, Weissmann C, Golovyashkina N, Sebo-Lemke Z, Drewes $\mathrm{G}$, Gerke $\mathrm{V}$, et al. The frontotemporal dementia mutation R406W blocks tau's interaction with the membrane in an annexin A2-dependent manner. J Cell Biol. 2011;192(4):647-61. doi:10.1083/jcb.201007161.

53. Reynolds CH, Garwood CJ, Wray S, Price C, Kellie S, Perera T, et al. Phosphorylation regulates tau interactions with Src homology 3 domains of phosphatidylinositol 3-kinase, phospholipase Cgamma1, Grb2, and Src family kinases. J Biol Chem. 2008;283(26):18177-86. doi:10.1074/jbc. M709715200.

54. Roberson ED, Halabisky B, Yoo JW, Yao J, Chin J, Yan F, et al. Amyloid-beta/ Fyn-induced synaptic, network, and cognitive impairments depend on tau levels in multiple mouse models of Alzheimer's disease. J Neurosci. 2011; 31(2):700-11. doi:10.1523/JNEUROSCI.4152-10.2011.

55. Chevalier-Larsen E, Holzbaur EL. Axonal transport and neurodegenerative disease. Biochim Biophys Acta. 2006;1762(11-12):1094-108. doi:10.1016/j. bbadis.2006.04.002.

56. Tank EM, Rodgers KE, Kenyon C. Spontaneous age-related neurite branching in Caenorhabditis elegans. J Neurosci. 2011;31(25):9279-88. doi:10.1523/ JNEUROSCI.6606-10.2011.

57. Hirokawa N, Noda Y, Tanaka Y, Niwa S. Kinesin superfamily motor proteins and intracellular transport. Nat Rev Mol Cell Biol. 2009;10(10):682-96. doi:10. 1038/nrm2774.

58. Kumar J, Choudhary BC, Metpally R, Zheng Q, Nonet ML, Ramanathan S, et al. The Caenorhabditis elegans Kinesin-3 motor UNC-104/KIF1A is degraded upon loss of specific binding to cargo. PLoS Genet. 2010;6(11): e1001200. doi:10.1371/journal.pgen.1001200.

59. Arimoto M, Koushika SP, Choudhary BC, Li C, Matsumoto K, Hisamoto N. The Caenorhabditis elegans JP3 protein UNC-16 functions as an adaptor to link kinesin-1 with cytoplasmic dynein. J Neurosci. 2011;31(6):2216-24. doi: 10.1523/JNEUROSCI.2653-10.2011.

60. Koushika SP, Schaefer AM, Vincent R, Willis JH, Bowerman B, Nonet ML. Mutations in Caenorhabditis elegans cytoplasmic dynein components reveal specificity of neuronal retrograde cargo. J Neurosci. 2004;24(16): 3907-16. doi:10.1523/JNEUROSCI.5039-03.2004.

61. Henriquez JP, Cross D, Vial C, Maccioni RB. Subpopulations of tau interact with microtubules and actin filaments in various cell types. Cell Biochem Funct. 1995;13(4):239-50. doi:10.1002/cbf.290130404.

62. Fulga TA, Elson-Schwab I, Khurana V, Steinhilb ML, Spires TL, Hyman BT, et al. Abnormal bundling and accumulation of F-actin mediates tauinduced neuronal degeneration in vivo. Nat Cell Biol. 2007;9(2):139-48. doi: $10.1038 /$ ncb1528.

63. Brunden KR, Trojanowski JQ, Lee VM. Evidence that non-fibrillar tau causes pathology linked to neurodegeneration and behavioral impairments. J Alzheimers Dis. 2008;14(4):393-9.

64. Marx J. Alzheimer's disease. A new take on tau. Science. 2007;316(5830): 1416-7. doi:10.1126/science.316.5830.1416.

65. Lasagna-Reeves CA, Castillo-Carranza DL, Sengupta U, Clos AL, Jackson GR, Kayed R. Tau oligomers impair memory and induce synaptic and mitochondrial dysfunction in wild-type mice. Mol Neurodegener. 2011;6:39. doi:10.1186/1750-1326-6-39.

66. Haass C, Selkoe DJ. Soluble protein oligomers in neurodegeneration: lessons from the Alzheimer's amyloid beta-peptide. Nat Rev Mol Cell Biol. 2007:8(2): 101-12. doi:10.1038/nrm2101.

67. Glabe CG. Common mechanisms of amyloid oligomer pathogenesis in degenerative disease. Neurobiol Aging. 2006;27(4):570-5. doi:10.1016/j. neurobiolaging.2005.04.017.

68. Caughey B, Baron GS, Chesebro B, Jeffrey M. Getting a grip on prions: oligomers, amyloids, and pathological membrane interactions. Annu Rev Biochem. 2009;78:177-204. doi:10.1146/annurev.biochem.78.082907.145410.

69. Walther DM, Kasturi P, Zheng M, Pinkert S, Vecchi G, Ciryam P, et al. Widespread proteome remodeling and aggregation in aging C. elegans. Cell. 2015;161(4):919-32. doi:10.1016/j.cell.2015.03.032.

70. Olzscha H, Schermann SM, Woerner AC, Pinkert S, Hecht MH, Tartaglia GG, et al. Amyloid-like aggregates sequester numerous metastable proteins with essential cellular functions. Cell. 2011;144(1):67-78. doi:10.1016/j.cell.2010.11.050.

71. Kenyon C, Chang J, Gensch E, Rudner A, Tabtiang R. A C. elegans mutant that lives twice as long as wild type. Nature. 1993;366(6454):461-4. doi:10. 1038/366461a0

72. Preuss U, Biernat J, Mandelkow EM, Mandelkow E. The 'jaws' model of taumicrotubule interaction examined in CHO cells. J Cell Sci. 1997;110(Pt 6):789-800.

73. von Bergen M, Barghorn S, Li L, Marx A, Biernat J, Mandelkow EM, et al. Mutations of tau protein in frontotemporal dementia promote aggregation of paired helical filaments by enhancing local beta-structure. J Biol Chem. 2001;276(51):48165-74. doi:10.1074/jbc.M105196200.

74. Kage-Nakadai E, Kobuna H, Funatsu O, Otori M, Gengyo-Ando K, Yoshina S, et al. Single/low-copy integration of transgenes in Caenorhabditis elegans using an ultraviolet trimethylpsoralen method. BMC Biotechnol. 2012;12:1. doi:10.1186/1472-6750-12-1.

75. Brenner S. The genetics of Caenorhabditis elegans. Genetics. 1974;77(1):71-94.

76. Gao S, Zhen M. Action potentials drive body wall muscle contractions in Caenorhabditis elegans. Proc Natl Acad Sci U S A. 2011;108(6):2557-62. doi: 10.1073/pnas.1012346108.

77. Jicha GA, Bowser R, Kazam IG, Davies P. Alz-50 and MC-1, a new monoclonal antibody raised to paired helical filaments, recognize conformational epitopes on recombinant tau. J Neurosci Res. 1997;48(2):128-32. doi:10.1002/(SICI)10974547(19970415)48:2<128:"AID-JNR5>3.0.CO;2-E.

78. Gottschalk A, Almedom RB, Schedletzky T, Anderson SD, Yates 3rd JR, Schafer WR. Identification and characterization of novel nicotinic receptorassociated proteins in Caenorhabditis elegans. EMBO J. 2005;24(14):2566-78. doi:10.1038/sj.emboj.7600741.

79. Tepper K, Biernat J, Kumar S, Wegmann S, Timm T, Hubschmann S, et al. Oligomer formation of tau protein hyperphosphorylated in cells. J Biol Chem. 2014;289(49):34389-407. doi:10.1074/jbc.M1 14.611368. 